University of Redlands

\title{
A Web GIS for the Economic Department of Highland, California
}

A Major Individual Project submitted in partial satisfaction of the requirements for the degree of Master of Science in Geographic Information Systems

by

Amanda Hutsel

Fang Ren, Ph.D., Committee Chair

Ruijin Ma, Ph.D.

August 2012 
A Web GIS for the Economic Department of Highland, California

Copyright (C) 2012

by

Amanda Hutsel 
The report of Amanda Hutsel is approved.

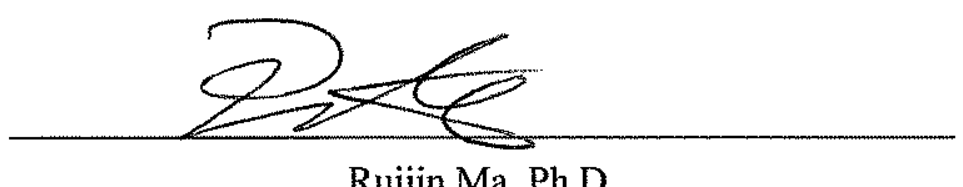

Ruijin Ma, Ph.D.

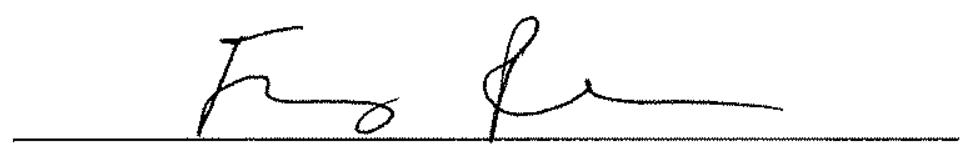

Fang Ren, Ph.D., Committee Chair

August 2012 



\section{Acknowledgements}

There are many people that I would like to thank for supporting me through this year. Thank you to my family and friends for your love and support through our many long phone calls. To Yusuf, thank you for all your time and patience while teaching me Flex. To Sara, thank you for being my gym and venting buddy. Thank you to my advisor, Fang, for constantly reminding me to not worry and "just do it". And to the rest of Cohort 20, I will always look back with fond memories of our barbeques by the pool and random front yard talks. 



\begin{abstract}
A Web GIS for the Economic Department of Highland, California
\end{abstract}

by

Amanda Hutsel

The Economic Department of Highland, California is responsible for records concerning the historically designated properties and grant money assisted homes. They were in need of a system to easily manage this information as the records were previously stored in multiple physical files that had to be sorted through when a question was asked. They also needed the system to be able to share their data with other Highland city departments.

This paper presents a Web GIS application as the solution to their problem. An ArcSDE Geodatabase was built to store the data and then a map document was published to the web using ArcServer 10. The web application was built using the ArcGIS API for Flex. It was created in such a way the user can easily view, query, and add to the records in the geodatabase. This application is also available to other Highland government departments to support a more efficient workplace. 



\section{Table of Contents}

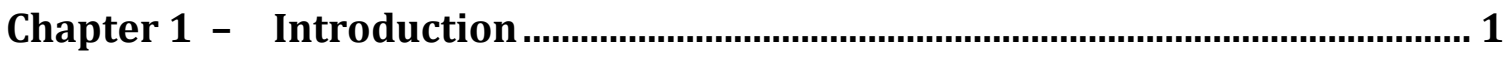

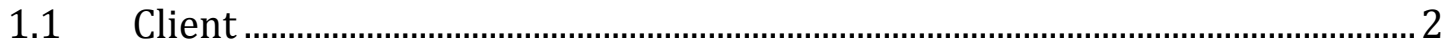

$1.2 \quad$ Problem Statement .............................................................................................

$1.3 \quad$ Proposed Solution ..............................................................................................

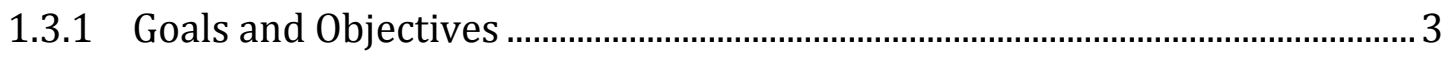

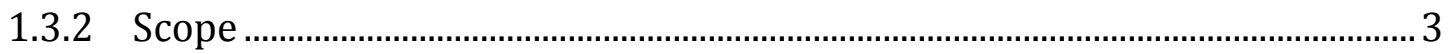

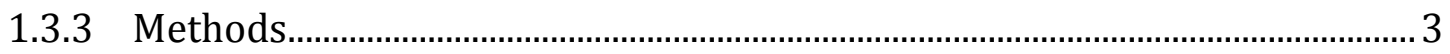

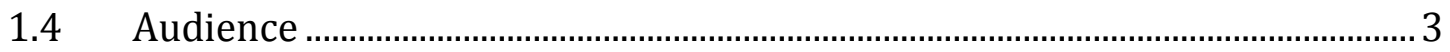

1.5 Overview of the Rest of this Report ................................................................... 4

Chapter 2 - Background and Literature Review................................................ 5

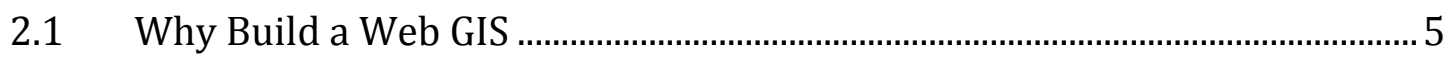

2.2 Building a Web GIS......................................................................................... 6

$2.3 \quad$ Geodatabase for a Web GIS ..................................................................................

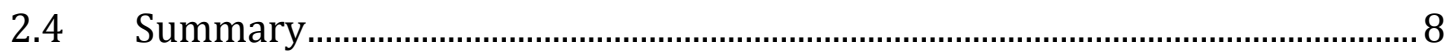

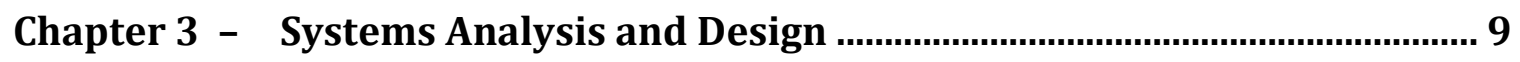

3.1 Problem Statement ..........................................................................................

3.2 Requirements Analysis ........................................................................

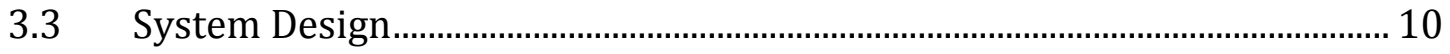

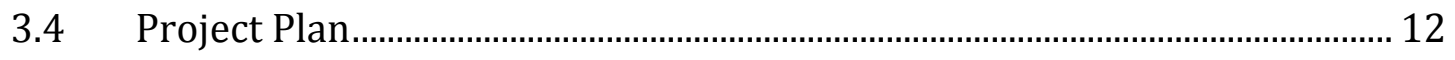

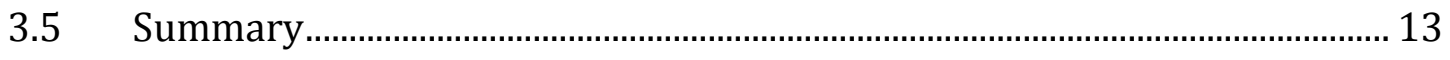

Chapter 4 - Database Design .......................................................................15 


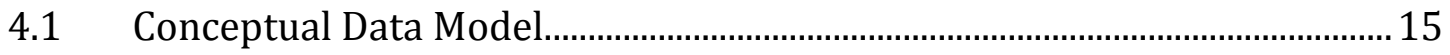

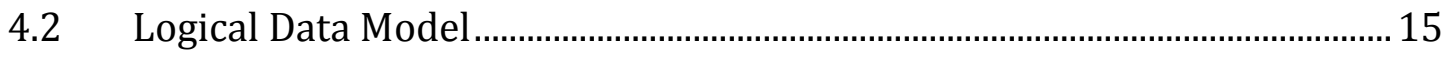

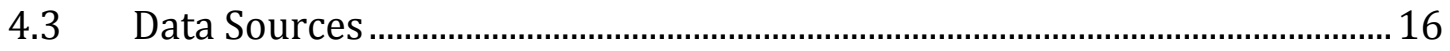

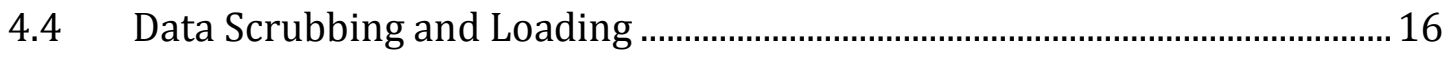

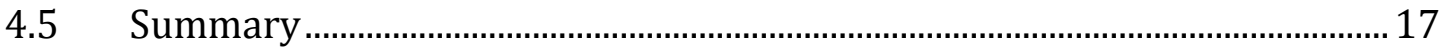

Chapter 5 - Implementation .................................................................................. 19

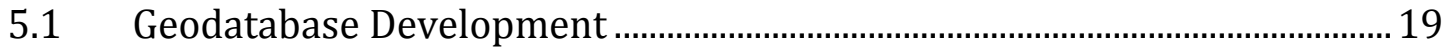

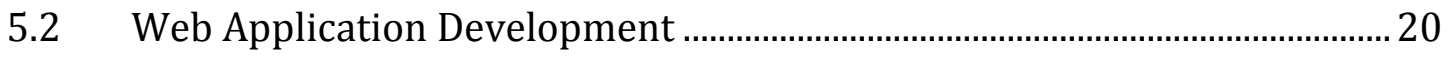

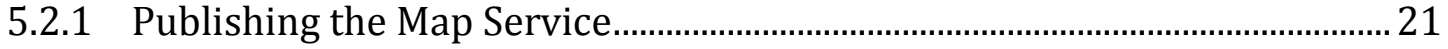

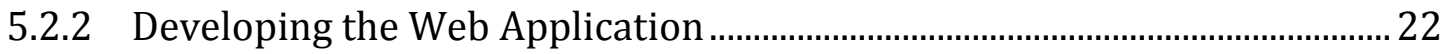

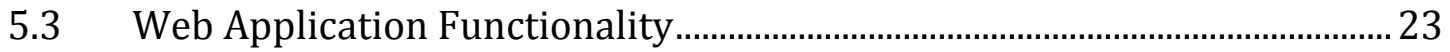

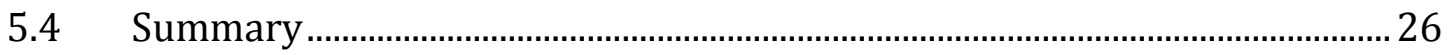

Chapter 6 - Use Cases ............................................................................................... 27

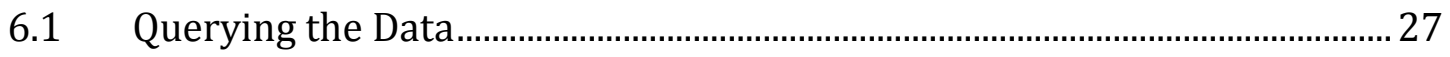

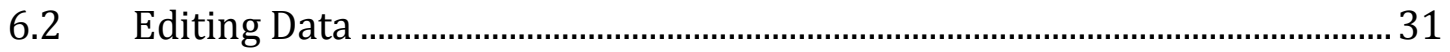

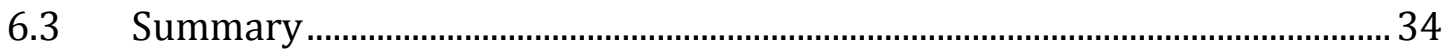

Chapter 7 - Conclusions and Future Work ............................................................ 35

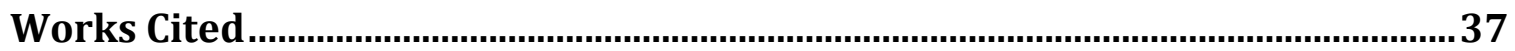

Appendix A. Web Map Application Code …....................................................... 39 


\section{Table of Figures}

Figure 1-1: Highland, CA area........................................................................... 1

Figure 2-1: Marine Irish Digital Atlas (Dwyer, 2011)........................................... 6

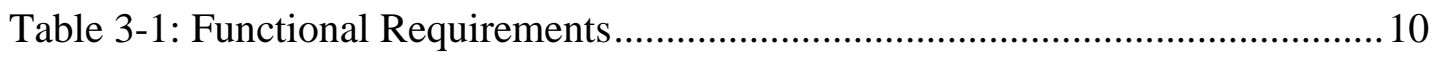

Table 3-2: Non-Functional Requirements ........................................................ 10

Figure 3-1: Example of geodatabase search functions (City of Riverside) ............... 11

Figure 3- 2: Example of attribute viewing on map (Esri, 2011) ............................ 12

Figure 4-1: Conceptual Data Model ............................................................... 15

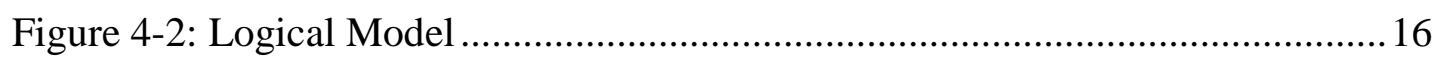

Figure 5-1: Map Document to be Published .......................................................20

Figure 5-2: Workflow for Building Application.............................................. 21

Figure 5- 3: Map Service Publishing Tools ..................................................... 21

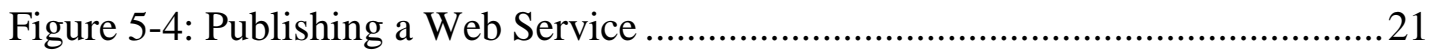

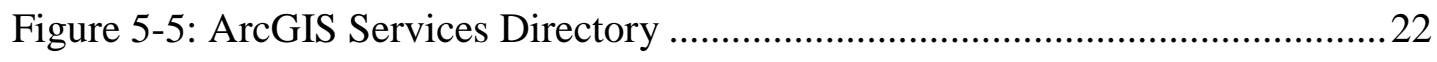

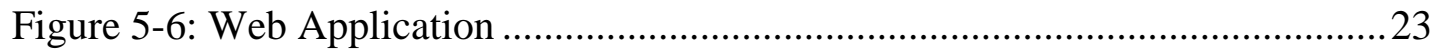

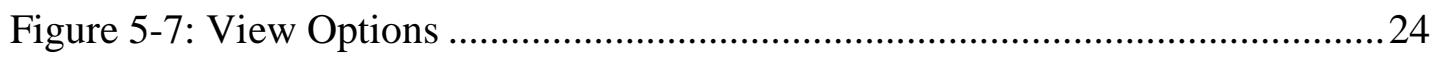

Figure 5-8: Search Function for Historical View (Left) and Grant View (Right) ..... 24

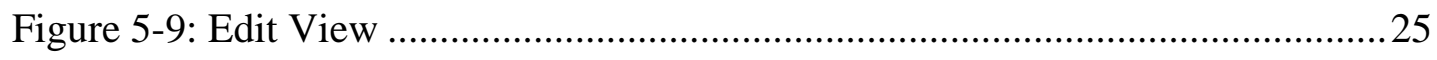

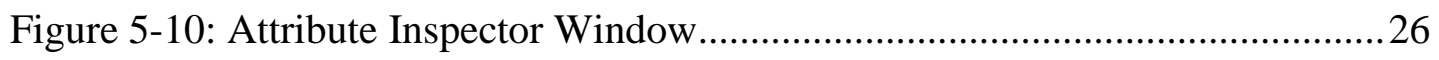

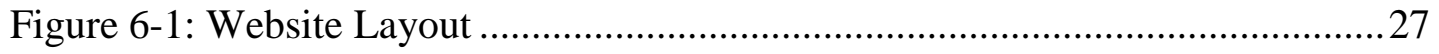

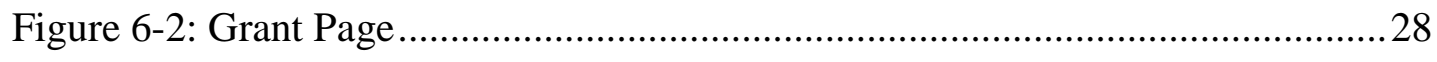

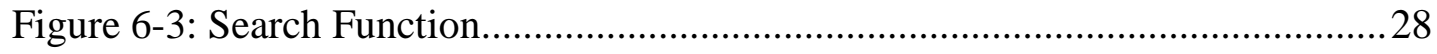




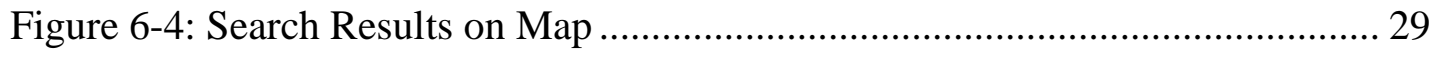

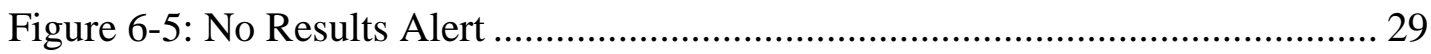

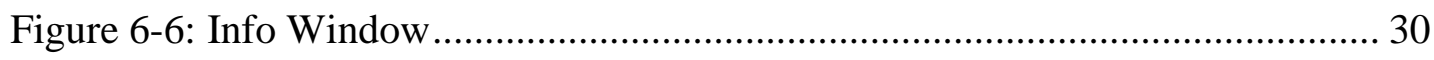

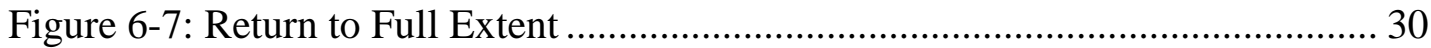

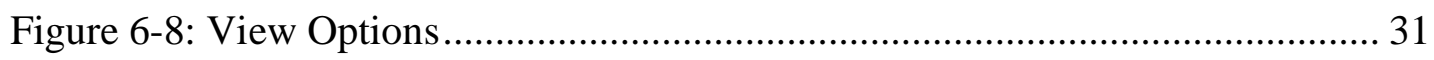

Figure 6-9: Finding Parcel for Editing Process .............................................. 31

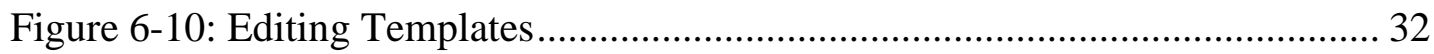

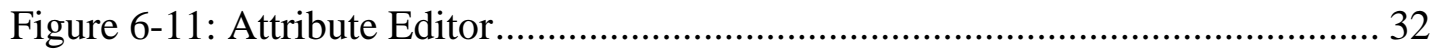

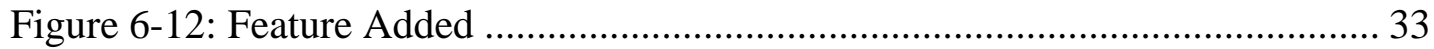

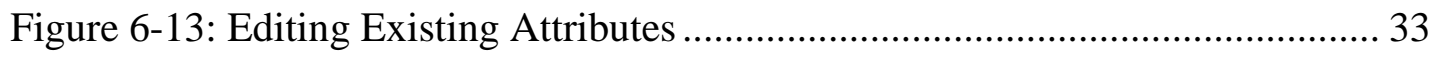

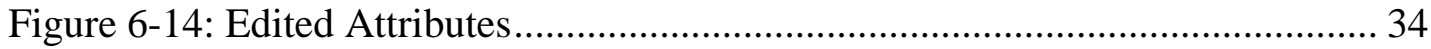




\section{List of Tables}

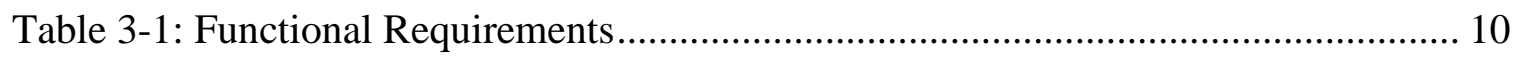

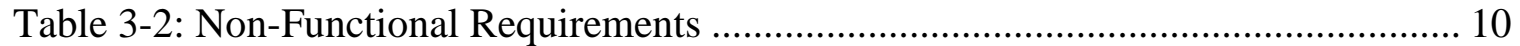





\section{List of Acronyms and Definitions}

$\begin{array}{ll}\text { API } & \text { Application Programming Interface } \\ \text { GIS } & \text { Geographic Information System } \\ \text { HTML } & \text { Hypertext Markup Language } \\ \text { IDE } & \text { Integrated Development Environment } \\ \text {.mxd } & \text { ArcGIS Map Document } \\ \text { MXML } & \text { Macromedia eXtensible Markup Language } \\ \text { SDK } & \text { Software Development Kit } \\ \text { URL } & \text { Universal Resource Locator }\end{array}$





\section{Chapter 1 - Introduction}

The City of Highland is a small city located in San Bernardino County approximately 66 miles east of Los Angeles (Figure 1-1). The town was founded in 1891 and was originally important in the citrus industry (City of Highland, 2007). Highland was officially incorporated in 1987 and today it is a city of approximately 53,000 people. The City still retains a small town atmosphere with many of the original buildings still standing.

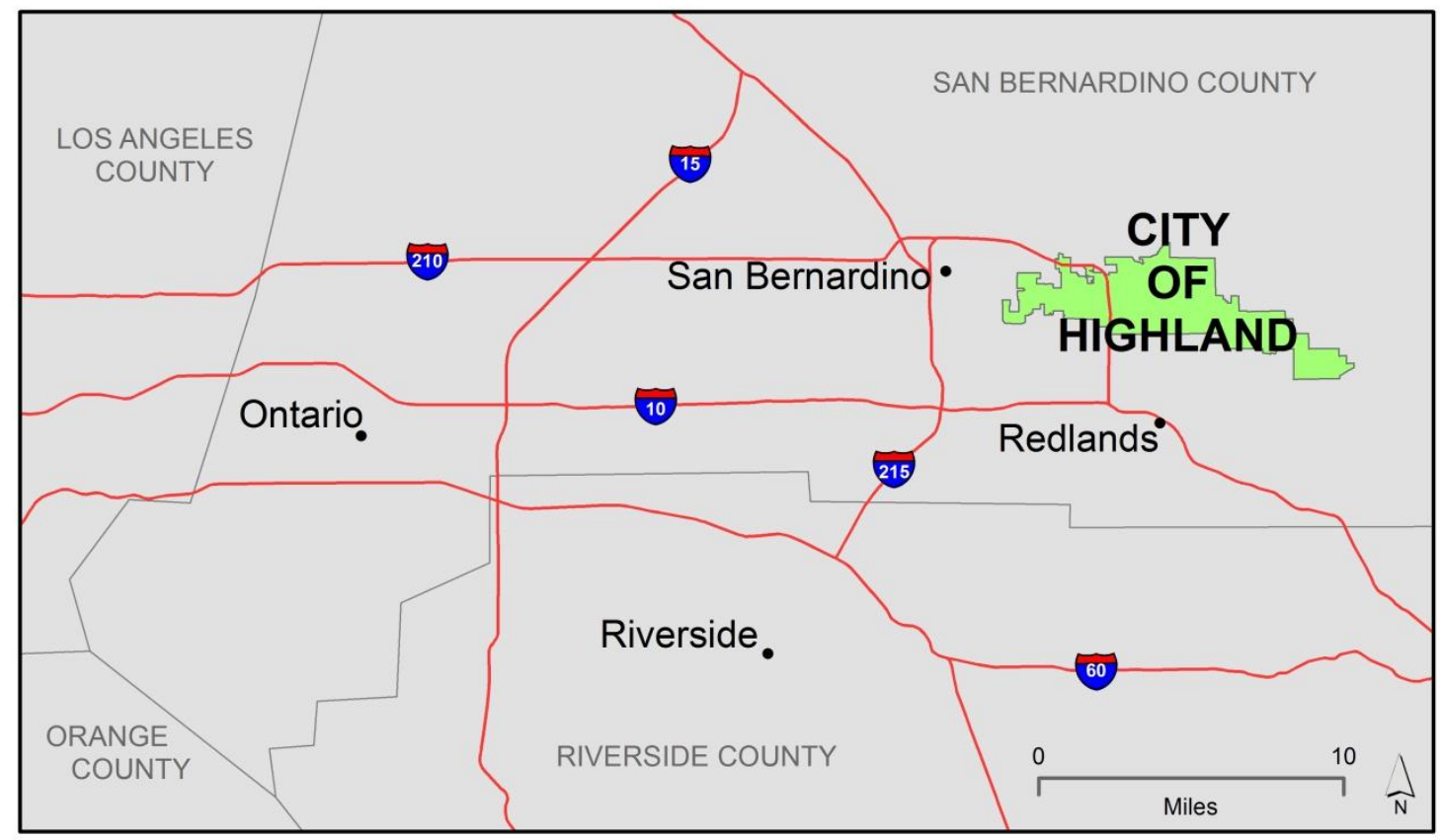

Figure 1-1: Highland, CA area

The Economic Department for the City of Highland is responsible for city planning and managing the historic data for the City. There were two areas in which the client wished to improve their data usability. The first of these concerns properties in the City that were assisted through the Neighborhood Initiative, Neighborhood Pride, and World Changers grant programs. These one-time grants of up to $\$ 25,000$ are available to low and moderate income, single-family residence owners to make repairs and improvements to their homes. These programs began in 1998 and since then have helped approximately 450 property owners. The other area of concern was historically designated properties in the City. These properties are historically designated by the National Register of Historic Places Program and the California Office of Historic Preservation and are listed in the National Register of Historic Places. There are currently 140 properties listed in Highland, both falling within the Historic District and outside the District.

These datasets resided in file cabinets and could only be accessed by other city departments through requests made to the Economic Department. The client for this project wished to have a system to allow easy access to their data and to display the data 
on a map. Thus, the goal of this project was to create a Web Geographic Information System (GIS) that would allow the city government staff to view and search records concerning grant program assisted homes and historically designated buildings. The system also needed to allow the client's staff to update and add to the records.

The following sections give an overview of the major components of this project. Section 1.1 and Section 1.2 introduce the client and her problem. Section 1.3 discusses the proposed solution, including the goals and objectives, the scope of the project, and gives an introduction to the methods used. The audience for this report is defined in Section 1.4, followed by an overview for the remainder of this report.

\subsection{Client}

The client for this project was Kim Stater, Economic Development Specialist for the City of Highland, California. Her responsibilities include maintaining the planning and historic data for Highland. She has also been a member of the Historic and Cultural Preservation Board since 1997. Her role in this project was providing the data on properties assisted by the Neighborhood Initiative and Neighborhood Pride grant programs and all historically designated properties. These datasets were provided in the form of Microsoft Excel spreadsheets.

\subsection{Problem Statement}

In the past, properties assisted by grant programs, as well as historic properties, were not contained in a database or represented spatially on a map. There was also no centralized source for all the different city government departments to consult before making decisions related to these properties. If information was needed, records, such as the Historical Architectural Reports and Planning Records, were available only in paper form. For example, the Housing Department may have issued a grant to a homeowner; as a result, the Building and Safety permit fees are waived for the work their contractor does. There needed to be a system to verify which homes had participated in the grant programs. In the case of a historically designated building, the Historic Board must approve any work requiring a permit. The Building and Safety Department needs to verify whether the home is designated in order to issue the required permit. The client requested a web application that would provide users with the location of the properties, as well as information about them. This application allows all the City's departments to view the records and the Economic Department the ability to edit and add to the records.

\subsection{Proposed Solution}

The proposed solution for the client was to develop a Web GIS application that the client and her staff could use to view, query, and update records in the Economic Department. The web application should be visible to all the City's government departments for use in decision making. 


\subsubsection{Goals and Objectives}

The main goal of this project was to develop an application that allows the City of Highland's government departments to easily view data concerning grant assisted homes and historically designated buildings. The application also had to be made available to the other departments in the City. To achieve this goal, a web application was developed to access a geodatabase containing the data provided by the client. A user can quickly find requested information, answer questions, and make decisions more efficiently.

The secondary goal was to design a database containing the data provided by the client. A user should be able to browse, query, edit, and add records to the database through the web interface. This should not require GIS knowledge or editing of the geodatabase through ArcGIS Desktop.

\subsubsection{Scope}

The scope of this project involved creating a web application that allows the user to query and display the data. It was built using Flash Builder and resides on the University of Redland's MS GIS web server. Esri's ArcGIS software was used to create a geodatabase of the locations of grant assisted properties and historically designated buildings. These datasets were provided by the client in the form of Microsoft Excel spreadsheets.

The database was designed to organize the data received by the client into a useable form. The web application allows a user to query this database and displays the information in an easy-to-use format. The application also allows the Economic Department to add and edit records.

\subsubsection{Methods}

A File Geodatabase was created using data provided by the client. When all the feature classes were complete, they were exported into an Enterprise ArcSDE Geodatabase. An ArcSDE Geodatabase allows for editing by users through a web application. The map document was created using this geodatabase in ArcMap 10 and was published to the web using ArcGIS Server 10. This published service resides on the University of Redland's MS GIS server. The web application was built using ArcGIS API for Flex version 2.5 and compiled using Adobe Flash Builder 4.6.

\subsection{Audience}

The audience for this report includes government employees with little to no knowledge of GIS or web programming. This report is geared toward these government employees who will be using the Web GIS so that they may better understand how the web application was created and how to use it to its full potential. The audience also includes those who might wish to recreate the web application for their own local government department. 


\subsection{Overview of the Rest of this Report}

The rest of this report is divided into six chapters. Chapter Two is a literature review including the topics of why a department should choose to use a Web GIS and the process and choices concerning building one. Chapter Three discusses the system analysis and design, including how the project was originally designed and how it changed over time. Chapter Four details the preparation of the data and subsequent design of the geodatabase. Chapter Five describes the implementation of the web application. Chapter Six contains use cases describing how this web application is being used. Chapter Seven concludes this report and offers ideas for the continuation of the project. 


\section{Chapter 2 - Background and Literature Review}

A Web GIS is a valuable way to bring GIS to the world and can be used in a wide variety of ways. This chapter will show the overall benefit and design of a Web GIS. Section 2.1 will discuss why a Web GIS should be chosen over a traditional GIS system. Section 2.2 will introduce several methods for developing a Web GIS application and what the benefits to each are. Geodatabase design will be discussed in Section 2.3.

\subsection{Why Build a Web GIS}

People have been using paper maps and mapping technology for centuries to disperse information and make decisions. The downside to these methods is the inability to easily share updated information. For paper maps, each time an addition is made to the data, a new map has to be made. Desktop mapping technology can solve this problem, making edits easier, but the information is not available to anyone else except the person using that software or application. Purchasing individual licenses for each employee to use mapping technology can be costly and time consuming.

With the increased use of the Internet in everyday life, a Web GIS can be a costsaving solution for sharing up-to-date spatial information throughout a company and is independent of operating systems (Mathiyalagan, Grunwald, Reddy, \& Bloom, 2005). A real world example of this can be found in Mathiyalagan, Grunwald, Reddy, and Bloom (2005) where the authors built an application so that the South Florida Water Management District, Army Corp of Engineers, and the Florida Environmental Protection Agency could access data for decision making concerning Florida's wetlands. The information managed by the Web GIS lead to a more efficient workplace by presenting the information in an easy-to-understand format in the form of a map (Anderson, 2005). In another study (Li, Xiong, \& Ou, 2011), researchers built a Web GIS for the public and scientific researchers to allow online access to sea ice data in the form of ice charts. The application allows users to access, explore, visualize, and analyze the sea ice data. It is both detailed enough for the scientist to perform complex analysis of historical ice data, and simple enough that the general public can browse and search information on ice condition changes. Another benefit of a Web GIS is the ability to link spatial and nonspatial data for better information visualization (Kraak, 2004). An example of this can also be found in Mathiyalagan, Grunwald, Reddy, and Bloom (2005) where they used soil, chemical, and biological properties, along with georeferenced point observations, to enable the government departments in Florida to visualize data customized for a specific topic in order to make informed decisions.

Finally, a Web GIS allows a map to become interactive and to function as a search engine (Kraak, 2004). The Marine Irish Digital Atlas (Dwyer, 2011), shown in Figure 21 , is an example of this functionality. They built a web application to search for various marine related data, such as beach or marina locations and the locations of many marine animals. When users find the information that they are looking for they can view it on the map, view associated metadata, and download the data to be used for analysis. 


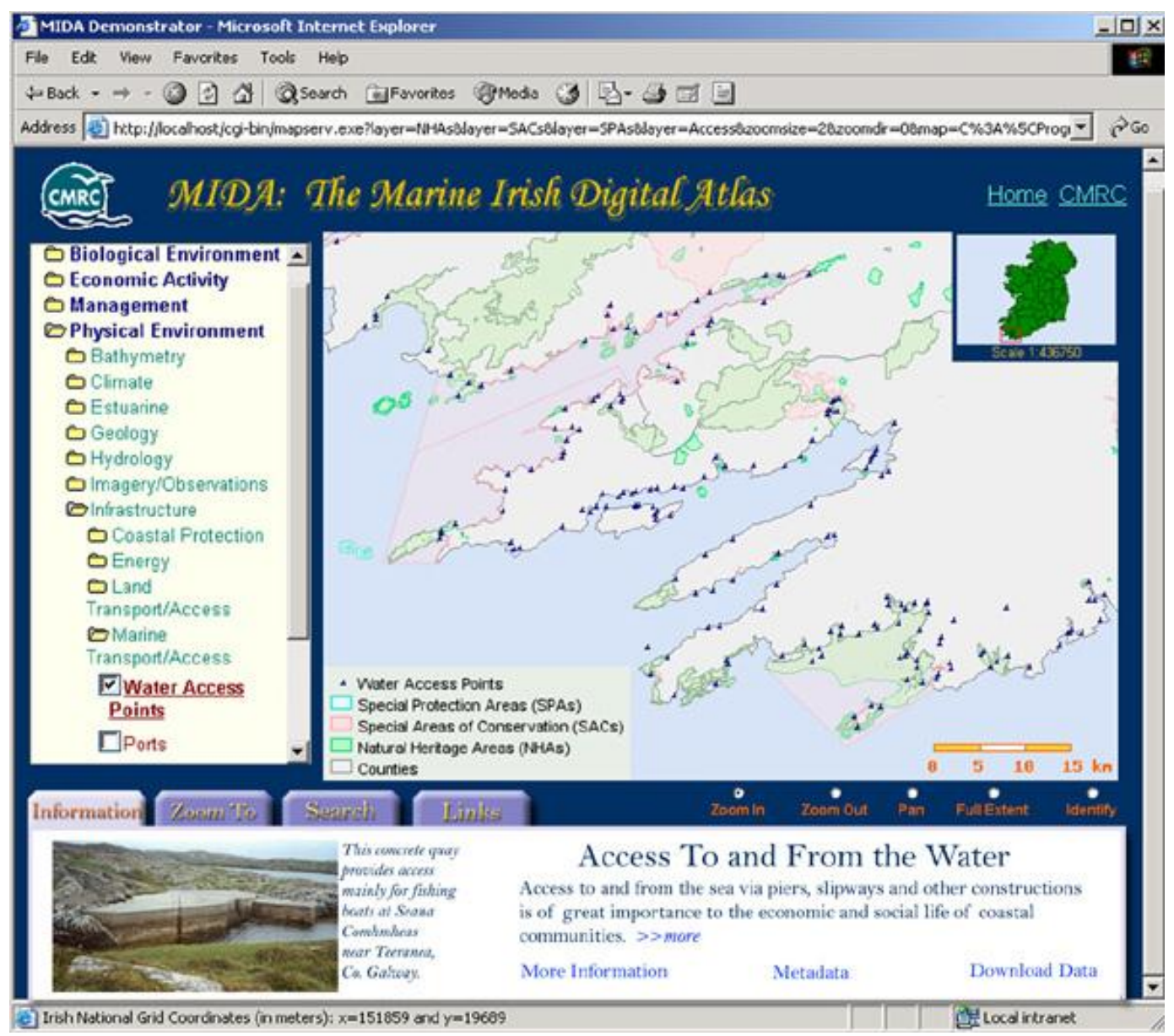

Figure 2-1: Marine Irish Digital Atlas (Dwyer, 2011)

\subsection{Building a Web GIS}

A web application can vary widely, from a simple map to a sophisticated interface with various spatial analysis functions (Adnan, Singleton, \& Longley, 2010). The interface can be built using different application programming interfaces (API) that will change the look and feel of the application. The choice of API depends on the programmer's preference and the user's needs. ArcGIS Online is a non-API option for sharing data for those who do not wish to program an application, although this option is very limiting. The JavaScript, Silverlight, and Flex APIs allow users to embed their own data with ArcGIS online basemaps into an application. Both allow users to search and edit features and attributes in their data (Fu \& Sun, 2011). These four Web GIS options and their individual benefits and drawbacks will be discussed further.

Esri's ArcGIS Online is a geoportal that "enables anyone to create, view, and use intelligent maps" (Intelligent web maps and ArcGIS Online, 2011) without needing ArcGIS software. Organizations can store their data in the "cloud" in the form of shapefiles or tables and allow others in their organization or the general public to access it. Users can then search for needed content and combine it with their own data to make a composite map (Fu \& Sun, 2011). The downside to ArcGIS Online is that it does not allow the user to update their database through the geoportal. There is also a file size 
limit of 2 gigabytes of data that can be uploaded with a standard license, making this a poor option for those with vast amounts of data.

ArcGIS API for JavaScript uses JavaScript and Hypertext Markup Language (HTML) to build web applications that can "accommodate sophisticated graphics and user interactions" (Fu \& Sun, 2011, p.97). Because the application is written using these languages, the user's web browser does not need any additional plug-ins. While applications using this API can be written in any standard text editor, this makes debugging the application more challenging (Brown, 2012) and applications may not work correctly on all browsers.

The ArcGIS API for Silverlight uses Extensible Application Markup Language and a sub-set of the .NET Framework. Applications are developed and debugged in Microsoft Visual Studio. The Silverlight API is used to create applications that are more visual complex than JavaScript. The API also includes many toolkits for editing and navigation to assist in application development (Esri, 2012). This can lead to a better user experience. To run applications built with Silverlight, the Silverlight plug-in installed in the browser and the Silverlight Application extension file must be installed on the web server containing the application (Lal, 2012).

ArcGIS API for Flex uses Macromedia eXtensible Markup Language (MXML) and ActionScript to build applications in Adobe Flash Builder. Due to the nature of the application, users must have the Adobe Flash Player plug-in installed with their browser. However, most browsers come with Flash Player pre-installed (O'Rourke, 2004). Flex is more expressive than JavaScript, with more flexible user interactions (Fu \& Sun, 2011) and is easier for novice web developers to use because it is developed inside an integrated development environment (IDE). The IDE provides a developer with interactive tools that make the design of an esthetically pleasing application straightforward (Baker \& Jayaprakash, 2009). Flex offers much flexibility in the interface design to accomplish this. Examples of this include the function of creating mashups, combining data from multiple web sources, and the many different ways to design the user experience. An Info Window can be customized to view attributes associated with a point on the map, but this also might be accomplished using the tool tip function. Another example is the layout of the application, which might be a simple map in the middle with a title or logo at the top, or could possibly contain multiple maps and/or boxes in which the user can find tools to help them navigate the data. The IDE also allows a developer to debug their application by providing error codes and messages. Another benefit is that an application built using the Flex API will run on all browsers without needing to be modified (Esri, 2010). Finally, Flex makes it easier to create Rich Internet Applications (RIAs), an application that has much of the same functionality of a desktop application. RIAs also allow increased productivity because the whole page does not have to reload every time the user interacts with the application (Baker \& Jayaprakash, 2009).

\subsection{Geodatabase for a Web GIS}

There are three types of geodatabases: personal, file, and ArcSDE. The choice of which geodatabase to choose depends on how it will be used, as each functions slightly differently. The personal geodatabase is meant for a single user and has a limited size of 2 gigabytes (Zeiler, 2010). The file geodatabase is not limited by size and can support more than one editor at a time, provided they are working on separate tables or feature 
classes (Zeiler, 2010). The ArcSDE geodatabase allows multiple editors to work on the same data at the same time, making it the choice for geodatabases built for Web editing purposes (Zeiler, 2010). ArcSDE also allows developers to easily publish services to the web using ArcGIS Server. Geodatabases designed using ArcSDE can be either versioned or nonversioned. Versioned databases are able to manage edits made by multiple users at the same time by temporarily storing them for review at a later date. The reviewer can look at what edits are made and accept changes to those that are correct or more up-todate. Nonversioned geodatabases, which are used by this project, do not store edits for review, but instead allow the user to directly edit the source data (Esri). This type is acceptable as only one person at a time will be editing the geodatabase.

\subsection{Summary}

Since the final product of this project will be used by those unfamiliar with GIS technology, a Web GIS application was chosen to be the solution to satisfy the client's needs. An ArcSDE geodatabase was developed for this project because it allows the editing of the geodatabase to be done through the Web GIS. This geodatabase does not need to be versioned because only one person will be editing at a time.

The ArcGIS API for Flex was chosen for this project from the different programming approaches to building Web GIS application. Flex was the optimal choice because of the flexibility in the design and customizability of the tools and interface. The client was also unsure of all users' web browser needs; therefore Flex was the optimal choice since it works on all browsers. 


\section{Chapter 3 - Systems Analysis and Design}

When working toward solving a problem, it is important to visualize how the idea can become a fully realized product. With regard to building a usable Geographic Information System (GIS), this involves looking fully into every aspect of the problem, the user's requirements, data availability, and the workflow for completing the project. This chapter details the system analysis and design, expanding on the proposed solution outlined in Section 1.3. The problem that was solved for the client is elaborated on in Section 3.1. Section 3.2 states the requirements that were made for this project. The system design is addressed in Section 3.3. The original project plan and how it changed with the progress of the project are discussed in Section 3.4.

\subsection{Problem Statement}

The two main issues the City of Highland faced were the ease of maintaining their data and the capability to share it easily across the other departments in the City. Highland's Economic Department is responsible for maintaining records involving homes improved with Neighborhood Initiative, Neighborhood Pride, and World Changers grant money and historically designated buildings. Prior to this project, these datasets resided in multiple file cabinets in the Economic Department office. The government staff needed to search through the cabinets every time a question was asked. For example, the Housing Department issues the grant and then the Building and Safety Departments need to know if the particular home is participating in a grant program in order to waive permit fees for the work the contractor does. All the data were located in the Economic Department and the other departments had to physically go to the Economic Department office to ask for the records. The client expressed the desire to have all data in one place and easily accessible to all the City's departments in order to facilitate the flow of information.

\subsection{Requirements Analysis}

The full requirements analysis of the system consisted of learning the functional and nonfunctional requirements for the end product. The functional requirements are determined by what the user wants the end product to be and to do. Non-functional requirements are system architecture qualities that are used to judge the success of the project, such as, does it work well on all operating systems and does it work in an acceptable time limit.

The functional requirements (Table 3-1) of this project are two-fold: there must be a geodatabase built from the data provided by the client, and a web application to view and modify this geodatabase. The client's functional requirements for the web application were to view, query, edit, and add to the data concerning the previously mentioned homes and buildings. The web application built to accomplish this requirement is accessed through the Internet from the University of Redland's MS GIS web server. There are two interfaces: one is accessible only to the Economic staff, the other is accessible by the other departments in the City of Highland. The interface for this application should work 
in any standard web browser, such as Firefox or Internet Explorer. Because of the private nature of the data, this system was not intended to be accessible by the general public.

Table 3-1: Functional Requirements

\begin{tabular}{|l|}
\hline Functional Requirements \\
\hline Web Application \\
\hline View and Query Data \\
\hline Edit Capabilities \\
\hline
\end{tabular}

Several non-functional requirements were considered for this project as well (Table 3-2). ArcGIS Server 10 was required to publish the web service and an ArcSDE Geodatabase was needed to allow editing of the data through the application. The web application needed to be intuitive because the users have no GIS experience. Other requirements for the completion of this project included ArcGIS Desktop for creating the map document, and the ArcGIS API for Flex version 2.5 and Adobe Flash Player for running the web application.

\section{Table 3-2: Non-Functional Requirements}

\begin{tabular}{|l|}
\hline Non-Functional Requirements \\
\hline ArcGIS Desktop and Server 10 \\
\hline ArcGIS API for Flex v2.5 \\
\hline Adobe Flash Player \\
\hline Intuitive Application \\
\hline
\end{tabular}

\subsection{System Design}

The design specification for the Web GIS system for the Economic Department of Highland contained the key components of a geodatabase: maps showing homes and buildings; and an interactive interface allowing users to view, query, edit, and add to records in the geodatabase. These were built based on the client's requirements outlined in the previous section.

Tables received from the client were imported into a File Geodatabase. These tables were slightly modified and converted into feature classes. They were then 
imported into an ArcSDE Geodatabase. This type of geodatabase allows for editing through the web application. The ArcSDE Geodatabase also allows for a large data storage space.

An ArcGIS map document (.mxd) file was created using the feature classes in the ArcSDE Geodatabase containing the homes improved using grant money, and the historically designated buildings. The .mxd also contained the parcel feature class that is used in the editing process described later. The .mxd was published to the web using ArcGIS Server. The web application was built using Adobe Flash Builder and the ArcGIS Flex API version 2.5. Several customized functions were required for the web application, including query of the records throughout the geodatabase, viewing the attribute information on the map, and editing the records. An example of a query function can be seen in Figure 3-1, which shows the City of Riverside's Historic District and Building lookup. An example from Esri's API for Flex resource page of viewing the attribute information on the map can be seen in Figure 3-2.

\section{Historic Districts \& Buildings}

\begin{tabular}{|l|}
\hline Historic Districts Home \\
\hline - Property \\
\hline - District \\
\hline - Property Viewer \\
\hline - Help \\
\hline
\end{tabular}

Search Database
- Select Property By One Or More Criteria:

Style:

Builder:

Architect:

Property Name:

Original Use:

Decade Built:

Designation Type:

District:
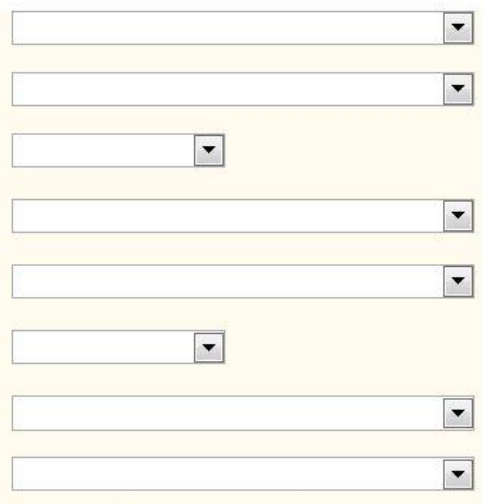

Results Clear All

Or Type an Address

Address:

GO

Type a street number and name.

Or Type an APN

APN:

Type an Assessor's Parcel Number.

Figure 3-1: Example of geodatabase search functions (City of Riverside) 


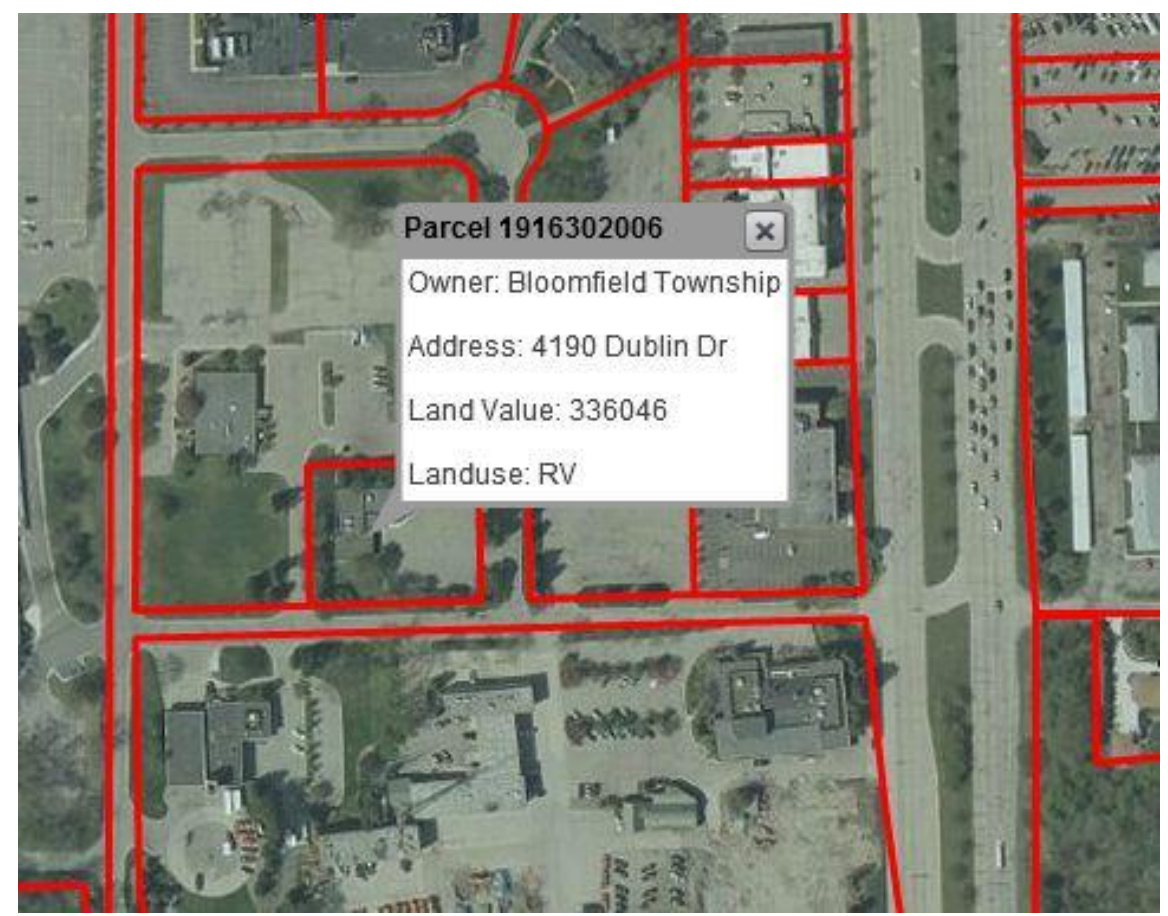

Figure 3- 2: Example of attribute viewing on map (Esri, 2011)

\subsection{Project Plan}

The original project plan consisted of six tasks: requirements analysis, data exploration, database design, application development, testing, and integration. During the requirements analysis phase the needs of the client were discussed and the type of system that would be built to meet these needs was determined. Due to the lack of GIS experience by the client, it was determined that a web application would best meet the requirements. While in the database design phase it was discovered that the database would need to be in the form of an ArcSDE Geodatabase. This type of Geodatabase allows multiple editors to be working on the same data and also allows for editing to take place through a web application. While the geodatabase was being built, several changes occurred to how the feature classes were created from the client's data. The first method of adding the features to a map was accomplished by geocoding using an address locator built from street data downloaded from the San Bernardino Associated Governments website (SANBAG GIS Data, 2011). Due to the addresses locator's lack of accuracy, not all features were placed in the correct location in which they physically appear. It was then decided to link each of the locations to the centroid of the corresponding parcel. This was accomplished by joining the parcel data to the client's data and then using the Make XY Event Layer tool. During the application development phase, the web application went through several changes in regard to the look and feel of the interface. Some of the changes, such as the layout, were strictly aesthetic; others, such as the movement between the view and edit modes, were functional in nature. The testing phase was a constant process during the application development phase as bugs were found and fixed. 


\subsection{Summary}

This chapter addressed the issues related to the project requirements, system design, and project plan. The fundamental requirements were to build a web application where the City staff, which does not have GIS technical skills, can use the interface to explore the data and update them in an intuitive environment. The original project plan was designed using these requirements and a system was built to fulfill their needs. Chapter Four will expand on the database design introduced in Section 3.4. 



\section{Chapter 4 - Database Design}

Database design was crucial to this project because it needed to be edited through the web application. This chapter details the steps that went into the database design process, from the conceptual model to how the data were made ready for use by the web application. Section 4.1 introduces the conceptual data model, including all the components and how they relate to one another. Section 4.2 details two logical data models, and states why the simplified model was chosen. Section 4.3 addresses the data sources, and Section 4.4 discusses how these data were made ready and loaded into the geodatabase.

\subsection{Conceptual Data Model}

Figure 4-1 shows the conceptual data model that includes major entities considered in the project and the relationships among these entities. A conceptual data model shows the component's relationships in a visual way, but does not show the relationships that are present in the actual database structure. In the data provided by the client, there are buildings that can be residential (homes) or commercial. Each building belongs in a parcel. There are owners of the homes and there are grants given to these homeowners to assist them in repairing and improving their homes. There are also historically designated buildings that are a mix of homes and commercial buildings. These buildings are given the historic designation by the National Register of Historic Places Program and the California Office of Historic Preservation.

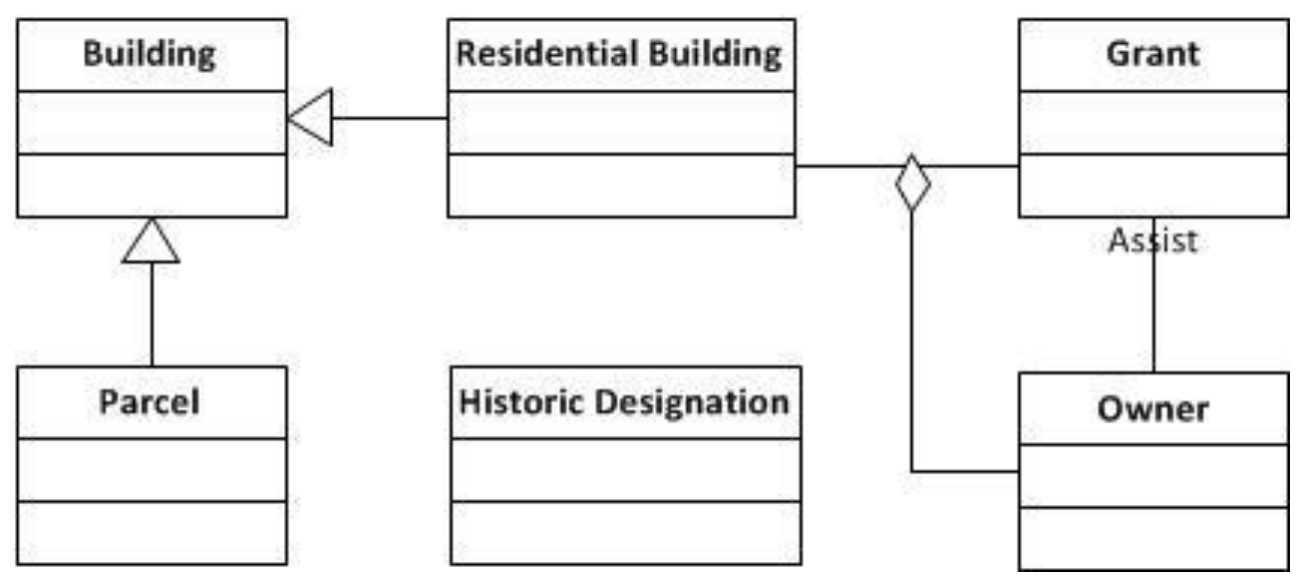

Figure 4-1: Conceptual Data Model

\subsection{Logical Data Model}

The logical model of the data presents the components from the conceptual model in the way they were applied in the geodatabase. There are many ways that the geodatabase could have been built. One method would require the combining of all data into a single table. Due to the different nature of the attributes found in the two datasets, this would have resulted in a large table with many null values. Instead, the method that was chosen 
separated the grant assisted homes and the historically designated buildings into different feature classes (Figure 4-2). This option was chosen for the simplicity of the design and to reduce the data storage space. Some buildings fell into both datasets, but since the data concerns different areas of interest, there was no need for them to be combined.

\begin{tabular}{|l|}
\hline \multicolumn{1}{|c|}{ Grant Assisted Home } \\
\hline+ +Owner : String \\
+Address : String \\
+Parcel Number : String \\
+Grant Program : String \\
+Grant Amount : Short \\
+Escrow Date : Date \\
+Reconveyance Date : Date \\
+Paid by Homeowner : Boolean \\
\hline
\end{tabular}

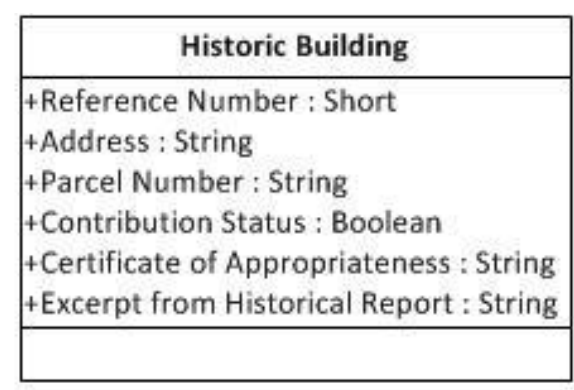

Figure 4-2: Logical Model

\subsection{Data Sources}

The datasets for this project were provided by the client in the form of two Microsoft Excel spreadsheets. One contained data concerning grant program assisted homes, the other with historically designated buildings. Each spreadsheet contained addresses and parcel numbers along with other attributes specific to the dataset. A complete parcel dataset was downloaded from the San Bernardino Associated Governments (SANBAG) GIS data website (SANBAG GIS Data, 2011).

\subsection{Data Scrubbing and Loading}

The data scrubbing and loading process began after all data had been received from the client or downloaded from online. Addresses and parcel numbers contained in the data provided by the client were checked for accuracy. Some addresses and parcel numbers were changed because they had been entered into the Excel spreadsheet incorrectly. The correct addresses and parcel numbers were found by the client in the original paperwork. Parcel numbers in the spreadsheets had the dashes removed to match the formatting of the parcel data downloaded from SANBAG (SANBAG GIS Data, 2011). The historical excerpt attribute in the historic data were spell checked and grammar checked. Spaces were removed from column headings to conform to ArcGIS data field standards. The spreadsheets were then imported into ArcGIS as tables and the text fields in the tables were changed to appropriated lengths to reduce data storage space. Initially the addresses were geocoded using street data from SANBAG. However, this was deemed to be too inaccurate, therefore the following process was used. The parcel data from SANBAG (SANBAG GIS Data, 2011) was clipped to the Highland city boundary and fields were added for the $\mathrm{X}$ and $\mathrm{Y}$ coordinates of the centroids of the parcel polygons. The parcel data and each of the tables received from the client were then joined with the parcel number being the key value. The Make X Y Event Layer tool was used to place the historic and grant program features in the centroid of the parcel in which they were 
found. Un-necessary data fields were deleted to reduce storage space and null values. These new feature classes were initially saved in an ArcGIS File geodatabase. When the final data scrubbing was complete, the three feature classes were exported to an ArcSDE Geodatabase.

\subsection{Summary}

This chapter discussed the steps that went into the database design phase of this project. What started as a conceptual model became a logically designed database that contained data from the client concerning historically designated buildings and grant assisted homes. The data sources and the data scrubbing process were then detailed, followed by how the datasets were used to create the ArcGIS geodatabase. 



\section{Chapter 5 - Implementation}

A custom web application for the viewing and editing of the City of Highland's

Economic Department's data was created for this project. This application allows the client to view, query, and edit data concerning grant assisted homes and historically designated buildings. This chapter discusses the process that was followed in the creation of the geodatabase (Section 5.1), how the data were published to the web using ArcGIS Server (Section 5.2.1), and how the web application was developed (Section 5.2.2).

\subsection{Geodatabase Development}

The database development was the first phase in the implementation of the project. The database was a critical component because it had to have the ability to be edited through a web application. The two datasets were received in the form of Microsoft Excel spreadsheets. Initial data scrubbing, such as modifying the field headings to remove spaces, spell checking, and verifying the addresses, was completed in Microsoft Excel. The spreadsheets were then imported into an ArcGIS File Geodatabase. There were two tables: one containing the grant assisted homes and the other containing historically designated buildings. To reduce file storage space, each of the text fields in the tables was shortened to an appropriated length. The city, state, and zip code fields were given default values so they did not have to be entered each time an edit is made since they are constant values within the City of Highland.

Parcel data from the San Bernardino Associated Governments (SANBAG GIS Data, 2011) were also imported into the geodatabase and X and Y coordinates of the centroid of the polygons were calculated and added to this feature class. These data were then joined to each of the two tables using the parcel number as the key value. The Make XY Event Layer tool was then used to create new feature classes from the grant and historic district tables. The Make X Y Event Layer tool creates a new point feature layer based on the $\mathrm{X}$ and $\mathrm{Y}$ coordinates in the source table. In doing so, each building and home was placed at the center of the corresponding parcel. The main reason for using the parcel data to define the locations of historical buildings and grant assisted homes was to improve the locational accuracy. In fact, the geocoding approach was attempted, but large location discrepancies were found for some buildings and homes.

The output of the Make X Y Event Layer tool was temporary. Each of the event layers were then exported into the ArcSDE Geodatabase, thus creating new feature classes with each feature in the correct location. The features were then symbolized so that they would show up on both street and imagery basemaps (Figure 5-1). 


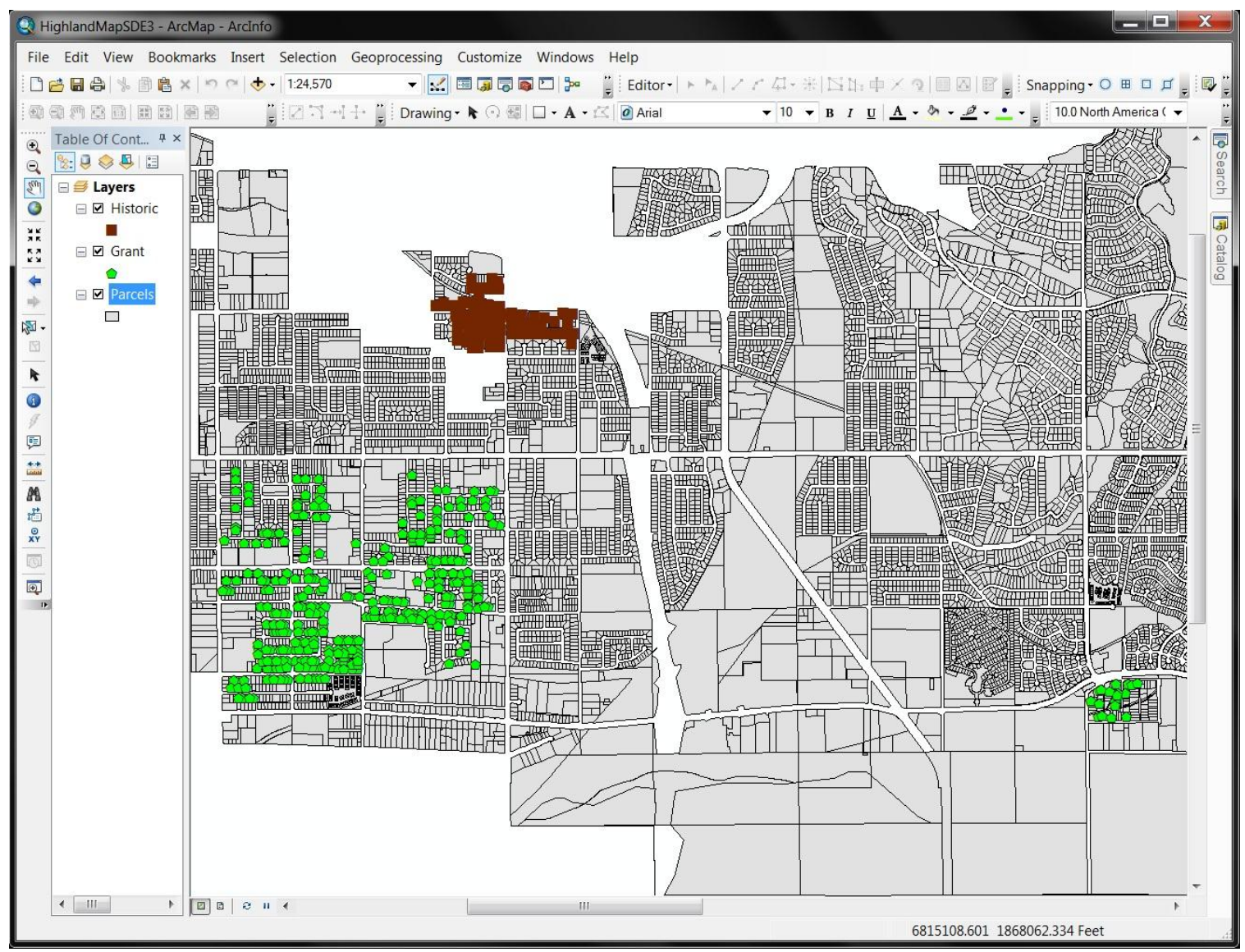

Figure 5-1: Map Document to be Published

The brown squares represent the historically designated buildings and properties and the green pentagons represent the grant assisted homes. These steps were completed in a File Geodatabase in ArcGIS Desktop 10. All feature classes were then imported into an ArcSDE Geodatabase. This type of geodatabase allows for multiple editors to be working on the same data at the same time and also allows feature classes to be edited through a web application. Each record in the Historic and Grant feature classes was given a global ID using the Add Global IDs tool in ArcGIS Desktop. The Global ID is similar to an Object ID except that it uniquely identifies each feature across the geodatabase. This number is automatically generated when a new feature is added. An ArcMap map document (.mxd) was then created containing the Historic, Grant, and Parcel feature classes (Figure 5-1).

\subsection{Web Application Development}

Once the ArcGIS map document (.mxd) displayed in Figure 5-1 was prepared, it was ready to be published to the web. The following sections explain the workflow that was followed in the creation of the web application for the Economic Department of Highland, CA (Figure 5-2). 


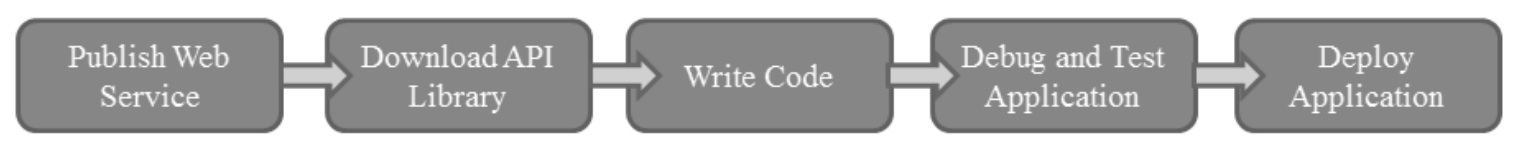

Figure 5-2: Workflow for Building Application

\subsubsection{Publishing the Map Service}

Tools found in ArcGIS Desktop 10 were used to publish the map document (.mxd) using ArcGIS Server 10 (Figure 5-3). The .mxd was first analyzed using the Analyze Map tool. This process looks for any errors that might prevent the published service from showing correctly. Some messages that might appear are that all the layers are drawn at all scale ranges. This could be an issue if the map has a small scale; all the features would show on the map even when zoomed to the fullest extent. For this .mxd, the scale range was not an issue as the map was a large scale map.

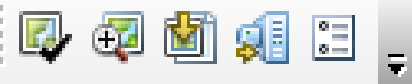

\section{Figure 5- 3: Map Service Publishing Tools}

The publishing process continued by using the Publish to ArcGIS Server tool, which opens the Publish to ArcGIS Server dialog box. Here the service is given a name and the folder into which the service is going to be published is specified. The next window allows the choice of what capabilities or functionalities the published service should have. The Mapping option is always checked, as this is the standard service that is used in all published maps. Because the geodatabase needed to have editing capabilities, it was published with the Feature Access option (Figure 5-4).

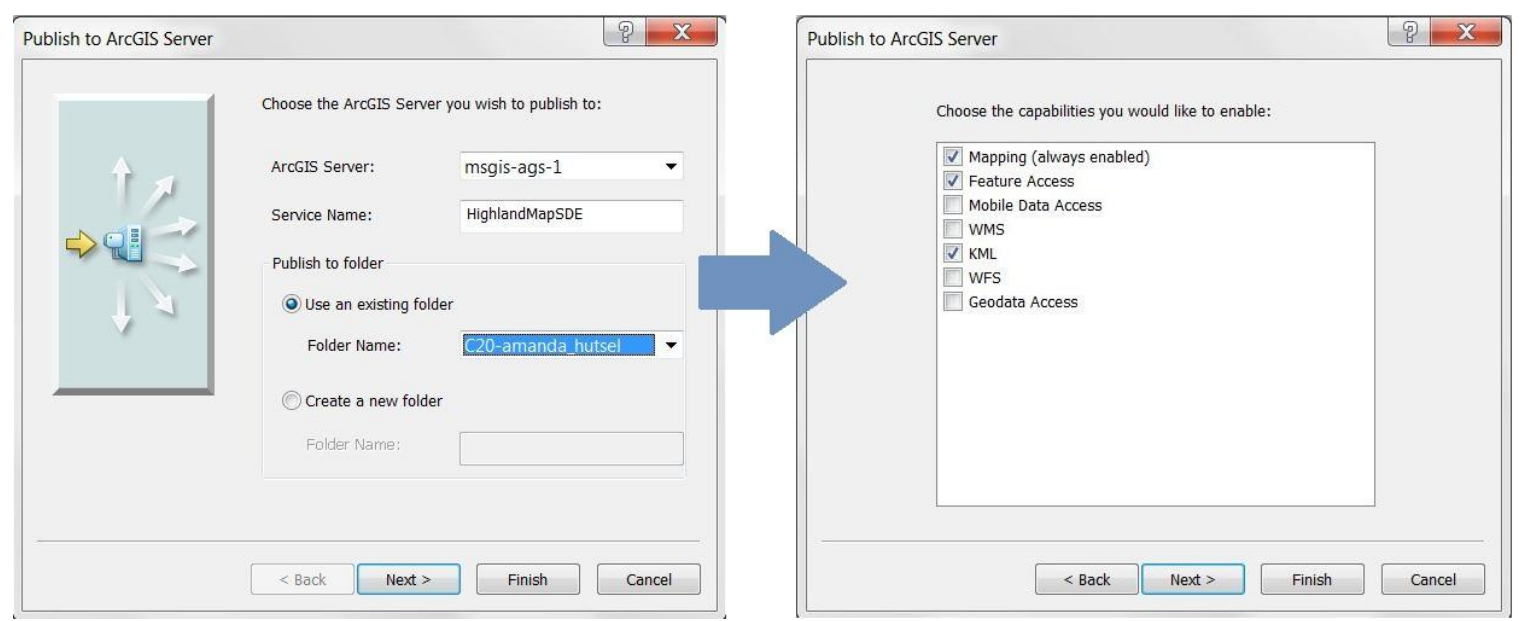

Figure 5-4: Publishing a Web Service

The Feature Access option links the geodatabase with the published service. When editing takes place, the web application informs the feature layer what edits have been made and adds them to the geodatabase (Esri, 2011). When the .mxd was published 
using ArcGIS Server, the services are found in the ArcGIS Services Directory under the folder that was chosen in the publishing process (Figure 5-5).

\begin{tabular}{l} 
ArcGIS Services Directory \\
\hline Home $>$ C20-amanda hutsel \\
\hline
\end{tabular}

\title{
Folder: C20-amanda_hutsel
}

\author{
Current Version: 10.03 \\ View Footprints In: Google Earth \\ Services: \\ - C20-amanda hutsel/Geometry (GeometryServer) \\ - C20-amanda hutsel/HighlandMapSDE3 (MapServer) \\ - C20-amanda hutsel/HighlandMapSDE3 (FeatureServer)
}

Supported Interfaces: REST SOAP Sitemap Geo Sitemap

Figure 5-5: ArcGIS Services Directory

The Directory shows all the published services and their layers that are on a particular server, as well as the capabilities and metadata of each layer. It also gives the ability to preview what each layer will look like on different Esri basemaps through the ArcGIS.com geoportal. When the published services were coded into the application, the Universal Resource Locator (URL) was used from the specific layers that were included in the map.

\subsubsection{Developing the Web Application}

Once the feature service was published, the development of the web application could begin. The application was built using the ArcGIS API for Flex, a software development kit (SDK) that is used for building rich Internet applications. Adobe Flash Builder was used as the integrated development environment (IDE) for this development phase. Flash Builder comes with built-in debugging tools to make the creation of an application easier. The debugging version of Flash Player was also used in the testing and debugging process.

The first step when building a Flex application is to download the ArcGIS API for Flex Library, available at the ArcGIS Resource page, and to import it into the library folder of the project workspace. This library allows access to functionality that is specific to the ArcGIS API for Flex and contains samples of Flex code. A new Flex project was then created in Flash Builder. Sample code available on the ArcGIS Resource page was used to begin the coding of basic functions and the layout of the interface. The coding of this application created a few custom tools for use by the Economic Department. These are discussed further in Section 5.3. Throughout the building of this application, multiple testing and debugging sessions were completed. The application was then deployed to 
the University of Redland's MS GIS web server for the term of one year. It was not put on the City of Highland's server because at the time of deployment, Highland did not have access to the ArcGIS Server or ArcSDE software.

\subsection{Web Application Functionality}

Different customized functions were coded for the web application (Figure 5-6). There were simple ones, such as switching basemaps, and more complex functions, such as multiple attribute querying and editing. The switching of basemaps was accomplished using a Button Bar with each button linked to an individual basemap. Two basemaps were used for this application, World Street Map and World Imagery, both from Esri's selection of standard basemaps, with the Street Map being the default.

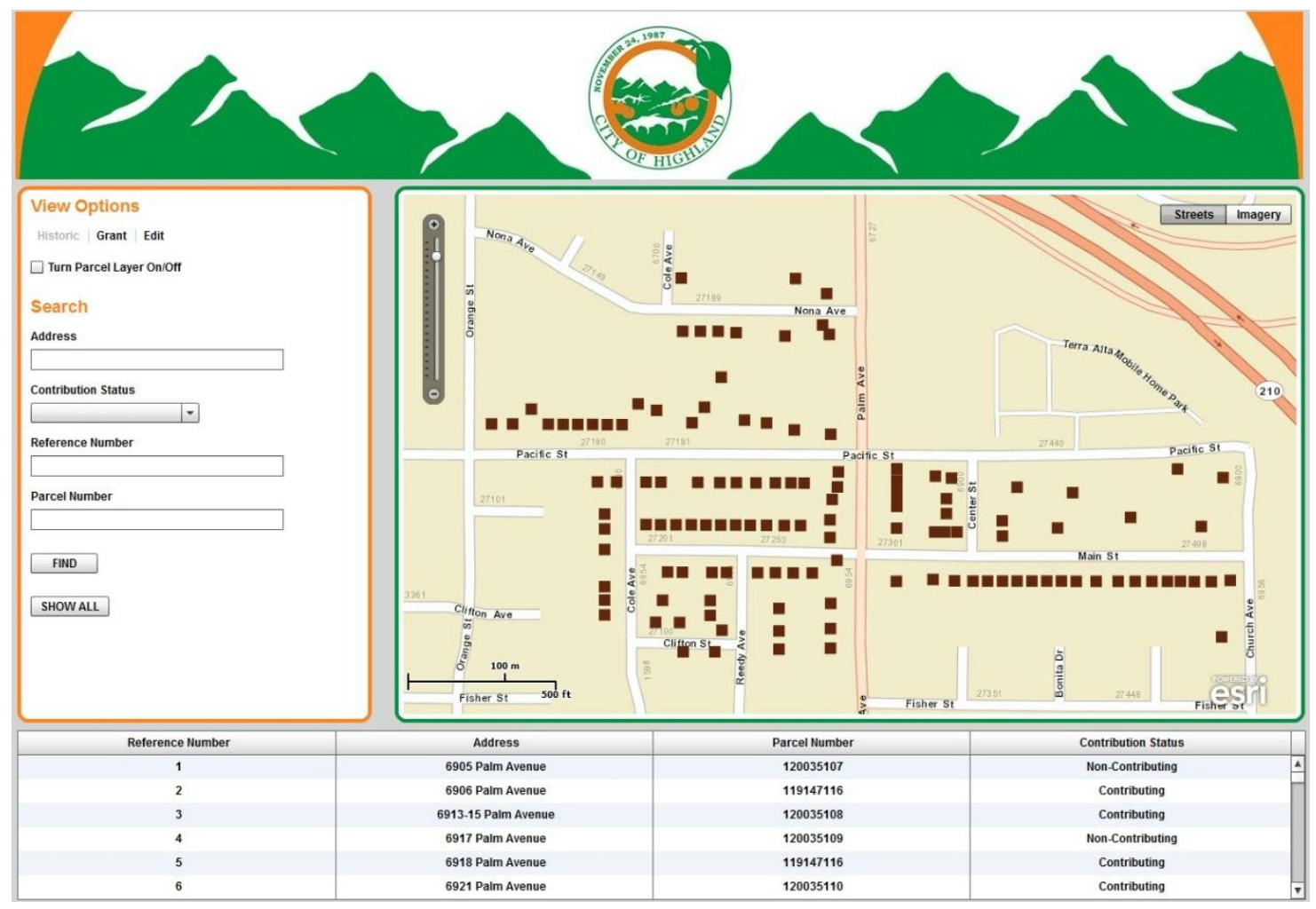

Figure 5-6: Web Application

Initially two applications were developed: one concerning the historically designated buildings, the other for the grant assisted homes. Each application had the same functionalities built into them in order to make the debugging of the code simple. The codes for each application were then combined into one application using the ViewStack method. Each of the views - Historic, Grant, or Edit - are contained within a $<$ NavigatorContent $>$ tag. This allows only one view to be visible at a time. The user then picks which view they would like to see using the Link Bar (Figure 5-8). The parcel layer was also made available to be viewed on each of the different views. A checkbox was coded so that the default is off but when checked the parcel layer is made visible. 


\section{View Options}

Historic | Grant | Edit

\section{Turn Parcel Layer On/Off}

Figure 5-7: View Options

The search functions for this application were more complex, as the user must have the ability to query for multiple attributes at the same time, or only part of an attribute. Figure 5-8 shows the attributes that can be searched for in the Historic view (left) and the Grant view (right).

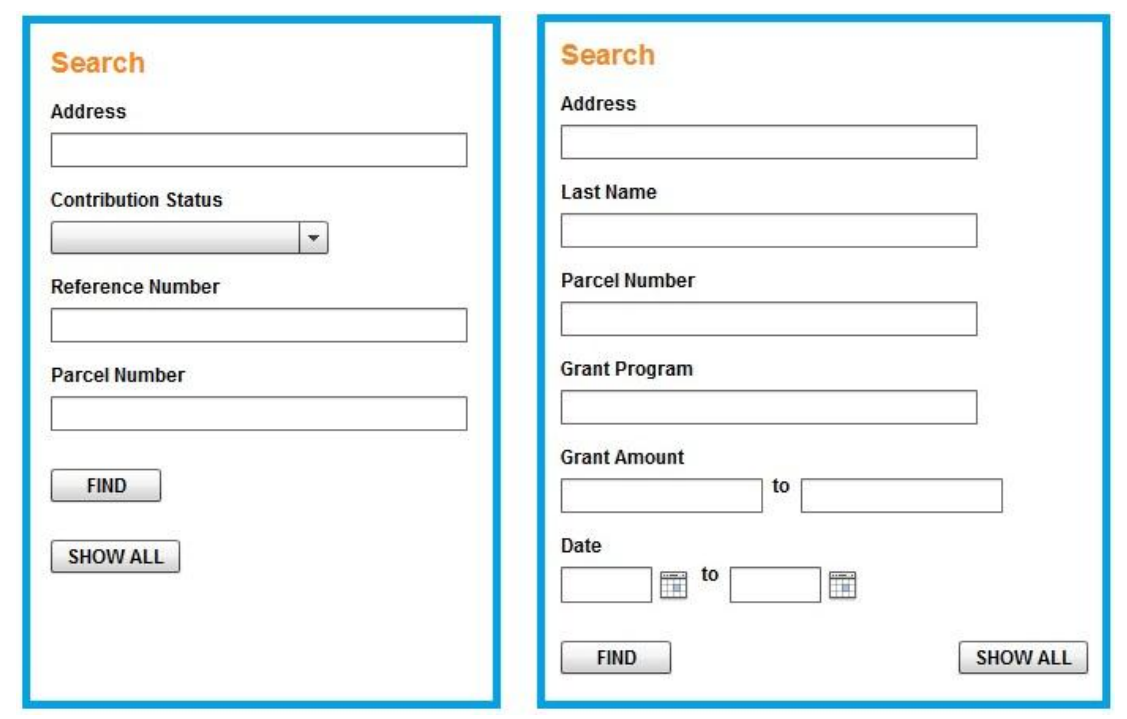

Figure 5-8: Search Function for Historical View (Left) and Grant View (Right)

There are three options to use when searching for features on a map using Flex: Find, Query, and Search Feature Layer. The Search Feature Layer was chosen because this allows the most flexibility in what can be searched for. A variable is created for the first of the searchable attributes then, using if statements, each of the attributes is added to the variable using the code str=str+ "and (attribute name)". A blank value is used if nothing is entered into that text box. For the historic layer, a user can search by an address, contribution status, reference number, and parcel number. For the grant layer, two more queries were included because the grant feature class contains more attributes. These include: address, last name, parcel number, and grant program. A user is also able to query for a date range. If nothing is entered in those fields, the function returns the entire time range of the data. There is also the ability to search for a range for the amount that was given in grant money. This function returns the results by making those found features the only ones to appear on the map. These search results must also appear in a table. This table was coded to show the attributes for each of the search results. Additionally, it also shows all records when the application is first opened. The function to return the results to the table was coded as part of the process that ends the search function. This section also returns error messages, if generated, and an alert box stating 
that no results were found. Code was also written that clears the form and shows all records by entering blank values for all searchable attribute fields. This function also returns the map to the original extent.

Editing functions are grouped into an Edit view (Figure 5-9). How the editing was to be accomplished changed many times in the process of creating the application.
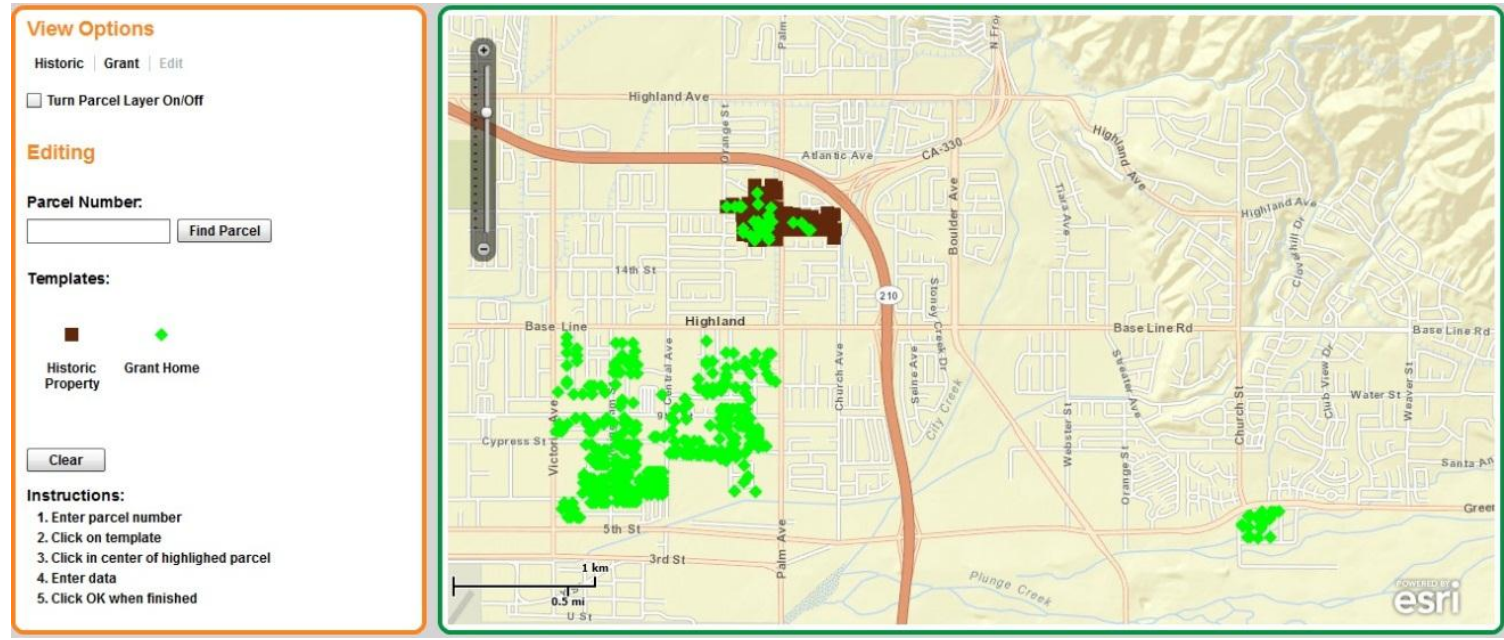

Figure 5-9: Edit View

The first option was to have a pop-up window opening on each of the two views Historic and Grant - which would have allowed the user to enter the attributes into a simple form. The window would have a SAVE button that saved the data to the geodatabase and added the point feature to the map using geocoding. However, the geocoding process was deemed unreliable when placing the feature in the correct location. Therefore the editing process was coded so that the user must complete multiple steps to add a new feature.

First, the user searches for a parcel using the Query method. This method was chosen because it returns the feature on the map in a separate graphic layer and therefore does not remove any of the other features showing on the map. In this case, the parcel that is queried will be zoomed into and highlighted on the map.

While still working with the feature classes in ArcDesktop templates were created for each of the layers that will be edited. The editing function was coded using the Default Editor component. This editor combines all the editing functionality into one component and saves new features and edits to the database automatically. It also pulls the templates that were created in ArcDesktop into the editing view and makes them selectable. The Default Editor component allows access to the editing version of the Attribute Inspector. This pop-up window reads the attributes in the feature layer and allows for the editing of them within text boxes (Figure 5-10). 


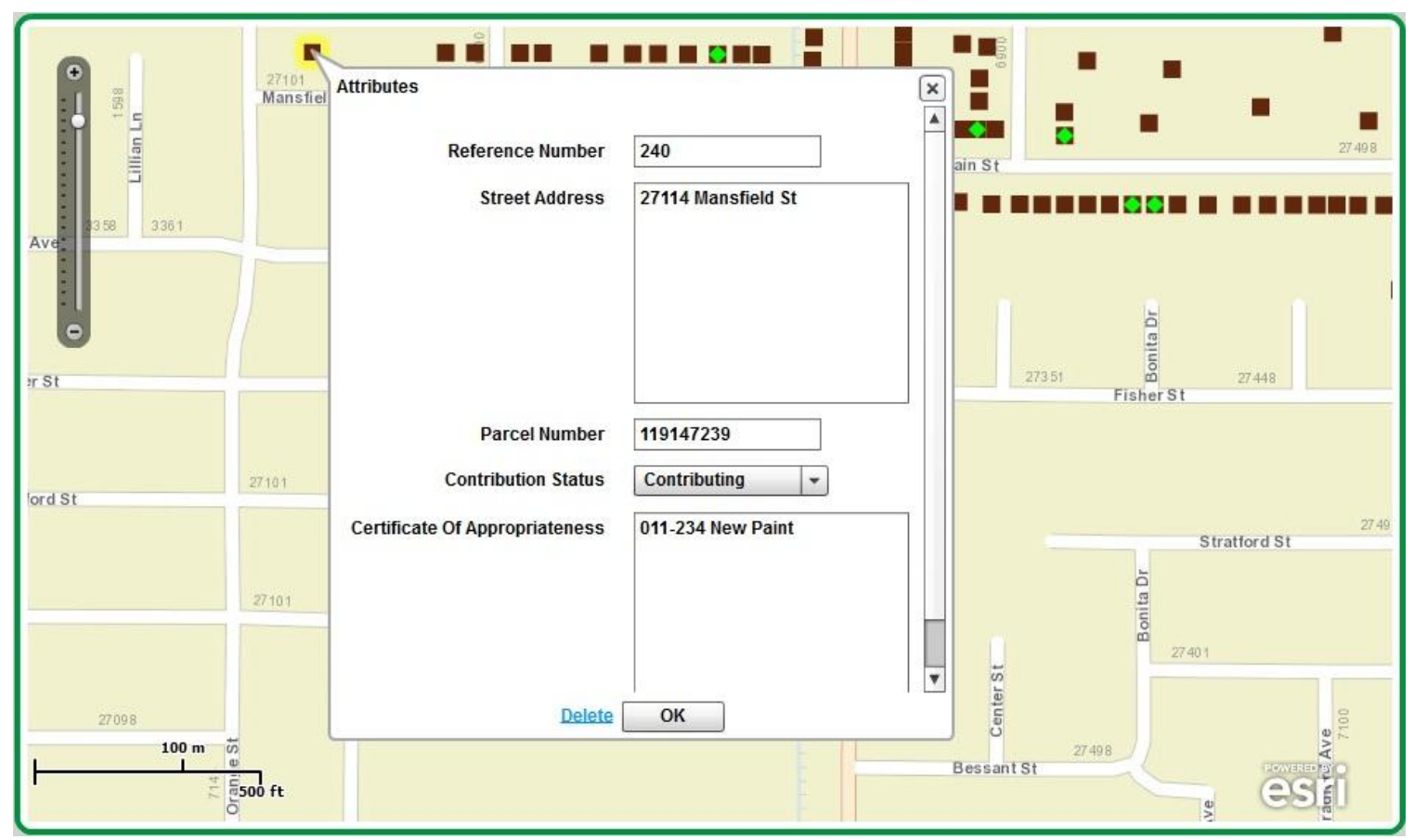

Figure 5-10: Attribute Inspector Window

The Attribute Inspector text boxes are directly linked to the corresponding attribute fields in the feature class. The date fields have a calendar added to them so that the user does not have to know the valid way to enter the date.

In the Historic layer, the field containing the contribution status was given a domain with three coded values since there are only three options: Contributing, Noncontributing, and Vacant Land. In the Attribute Inspector this field appears as a drop down menu so that there is less human error likely to occur. The Attribute Inspector also allows the user to edit the attributes of existing features. This editing process does not automatically assign the centroid of the parcel to the added feature as its spatial location. Instead, the location that the editor clicks on the map is the location recorded in the geodatabase for the added new feature. Therefore the editor should place the template marker inside the highlighted parcel on the map.

\subsection{Summary}

This chapter discussed the custom web application that was created for this project for the viewing and editing of the City of Highland's data. This application allows the client to view, query, edit, and add features belonging in the grant assisted homes and historically designated buildings datasets. It described the process that was followed in the creation of the web application for the City of Highland's Economic Department, from building the ArcSDE Geodatabase to how the map document was published, as well as the process of building the web application in Flash Builder using the ArcGIS API for Flex. The full code for this application can be found in Appendix A. 


\section{Chapter 6 - Use Cases}

There are two primary uses for the web application that was built for the Economic Department of Highland, CA. First, the application provides a way for the City of Highland's government departments to browse and quickly query data concerning grant assisted homes and historically designated buildings. It allows the client to add new records or to edit the existing records in the geodatabase without needing to have GIS knowledge or direct access through ArcGIS Desktop. This chapter presents two use cases: one for the Building and Safety Department in Highland who need to find out if a home has been given grant money (Section 6.1); the second use case is for the Economic Department who are adding a historically designated building to their database (Section 6.2). Several areas of the application interface are mentioned in this chapter, and they are labeled in Figure 6-1.

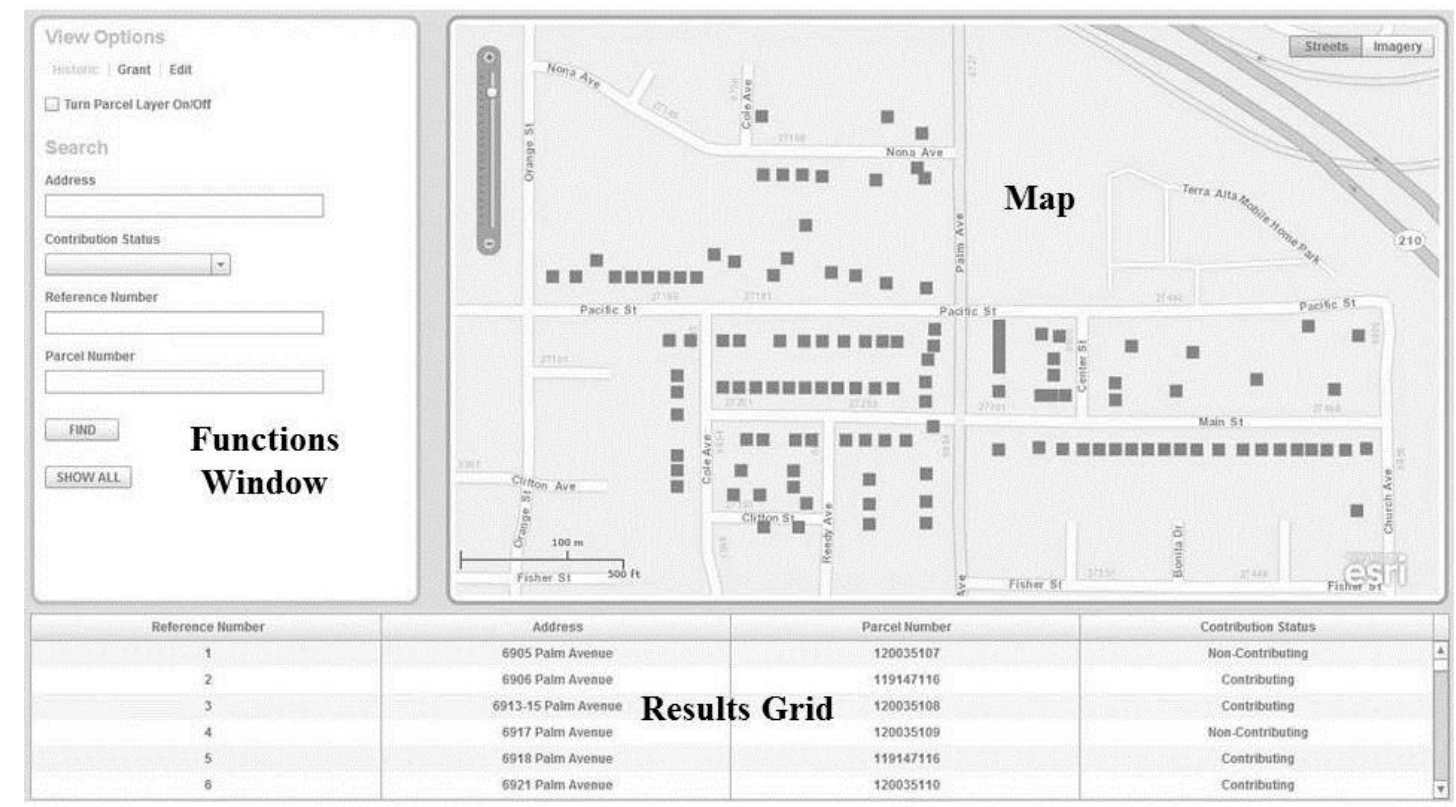

Figure 6-1: Website Layout

\subsection{Querying the Data}

Suppose the Building and Safety Department of Highland, CA needs to know if an owner has been given a Neighborhood Initiative, Neighborhood Pride, or World Changers grant by the Housing Department to improve or repair their home. If the home owner has been given a grant, the permit fees will be waived for the work his/her contractor does. To begin the process of searching for the specific households that have received the grant, a user will first open the application where the default page is the Historic view. To move to the Grant page (Figure 6-2), the user needs to switch by selecting the "Grant" option in the View Options (highlighted in the blue box in Figure 6-2) section of the Functions Window. 


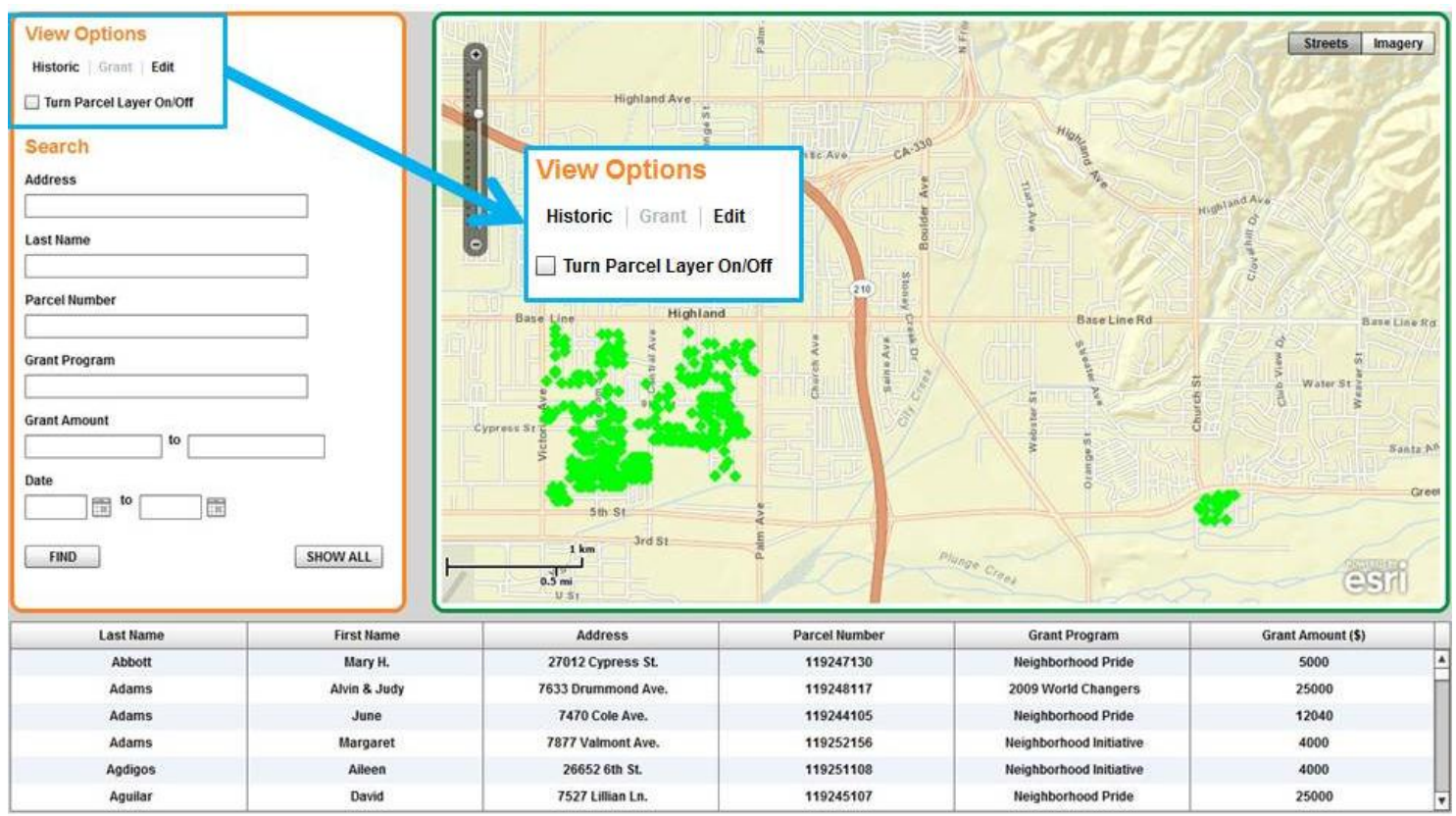

Figure 6-2: Grant Page

The Building and Safety Department would like to search for a home to determine if a grant has been given to the homeowner. The searchable attributes are found as text boxes in the Functions section. The user can enter in as many attributes as they like, narrowing the results. In this case, they are only using the homeowner's last name (highlighted in Figure 6-3). They will enter the name into the "Last Name" field and click the "FIND" button. This will execute the search function that was described in Chapter 5. The search results are displayed both on the map and in the Results Table below (Figure 6-4). If no results are found an alert shows (Figure 6-5).

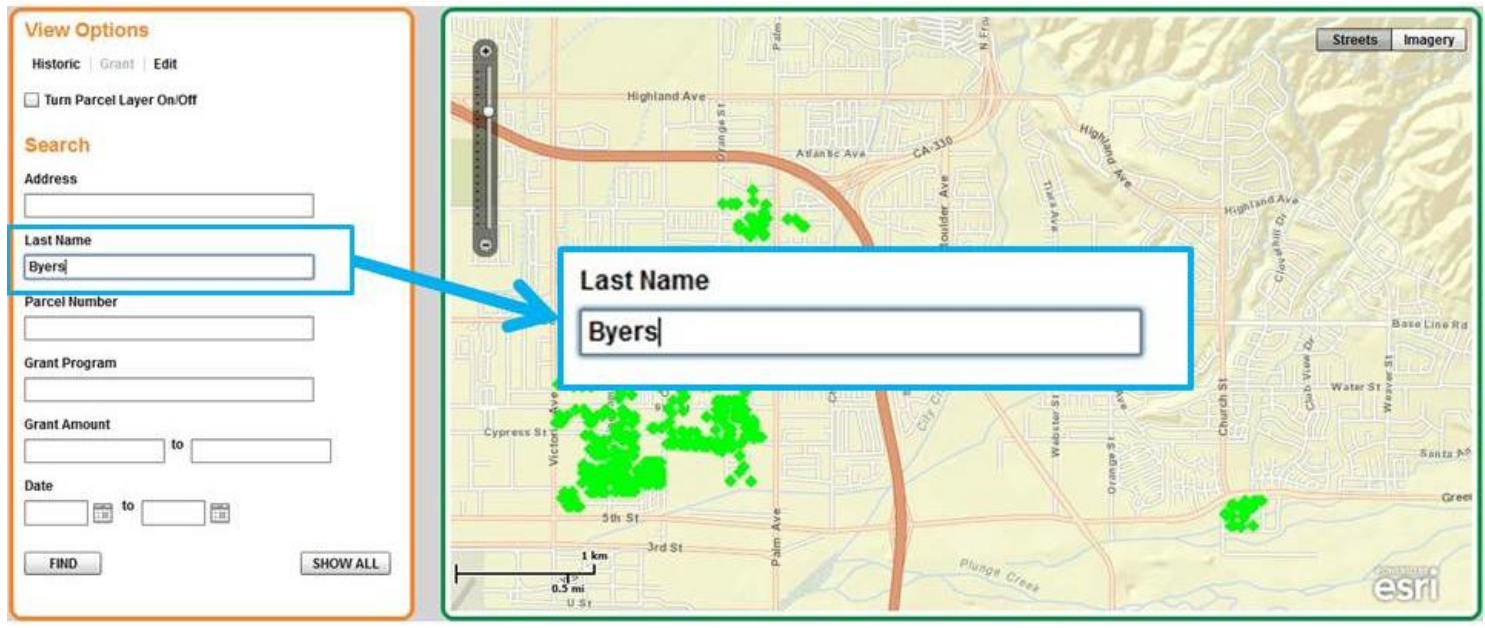

Figure 6-3: Search Function 


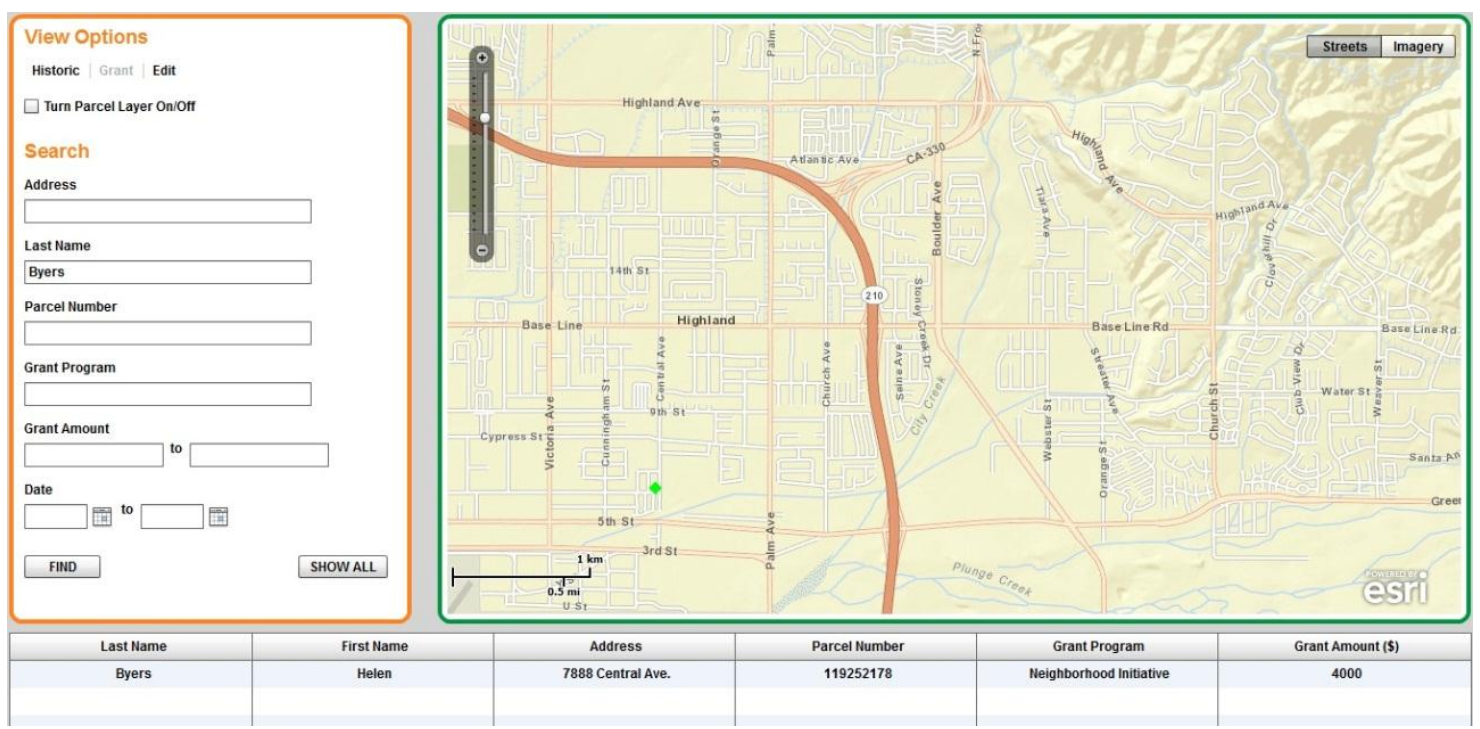

Figure 6-4: Search Results on Map

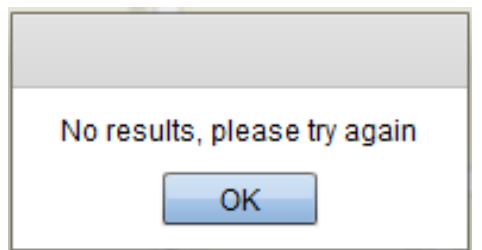

Figure 6-5: No Results Alert

To better view the found results they can zoom into the found features, as seen in Figure 6-6. Because some of the attributes do not show in the Results table, if the Department needs to know more they may view all the attributes by clicking on the point feature symbol. This will open an Info Window containing the attributes associated with this record (Figure 6-6). 


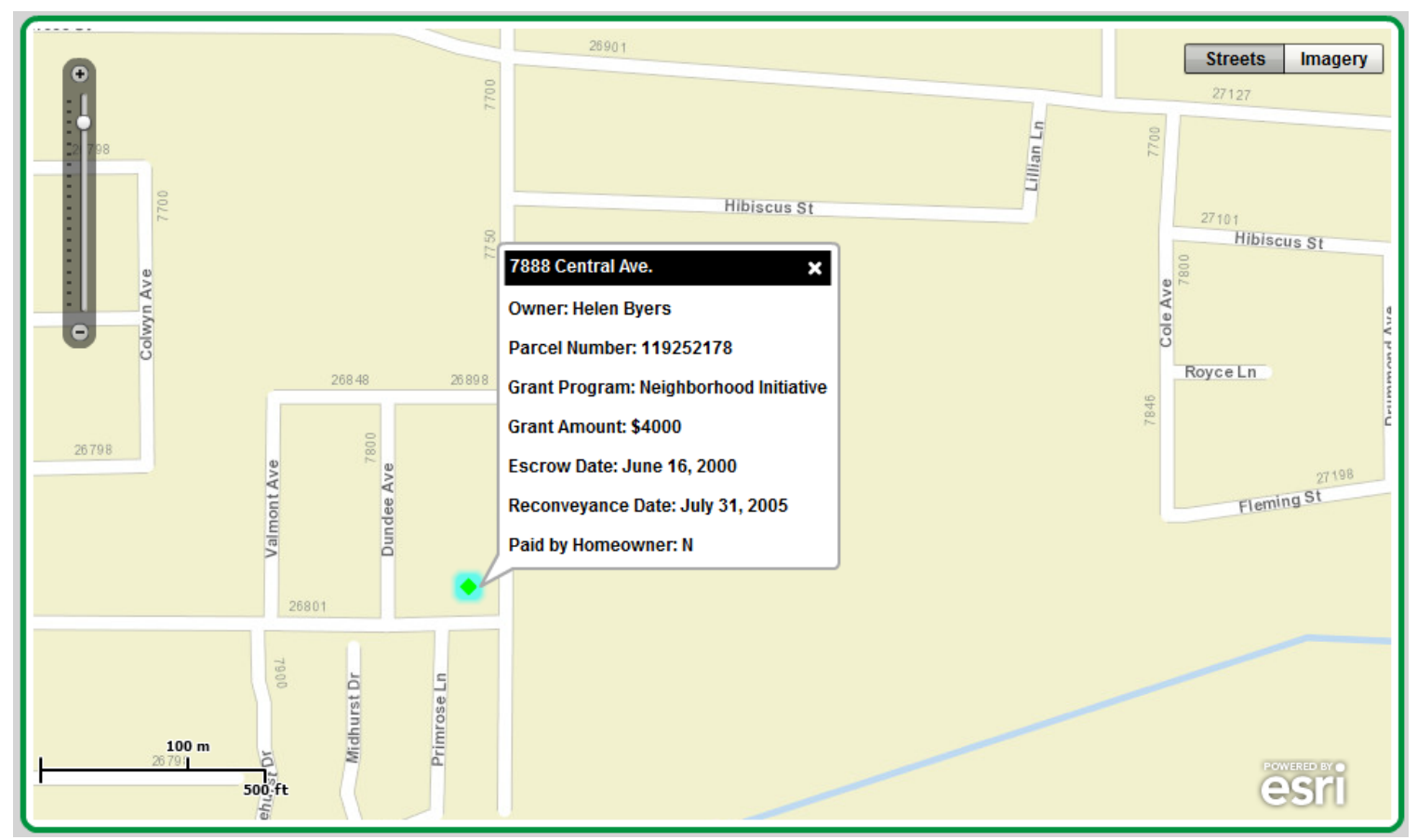

Figure 6-6: Info Window

If the Department would then like to search for another record, they can click on the "SHOW ALL" button in the functions window. This will clear all the found records and return the map to the default extent (Figure 6-7).

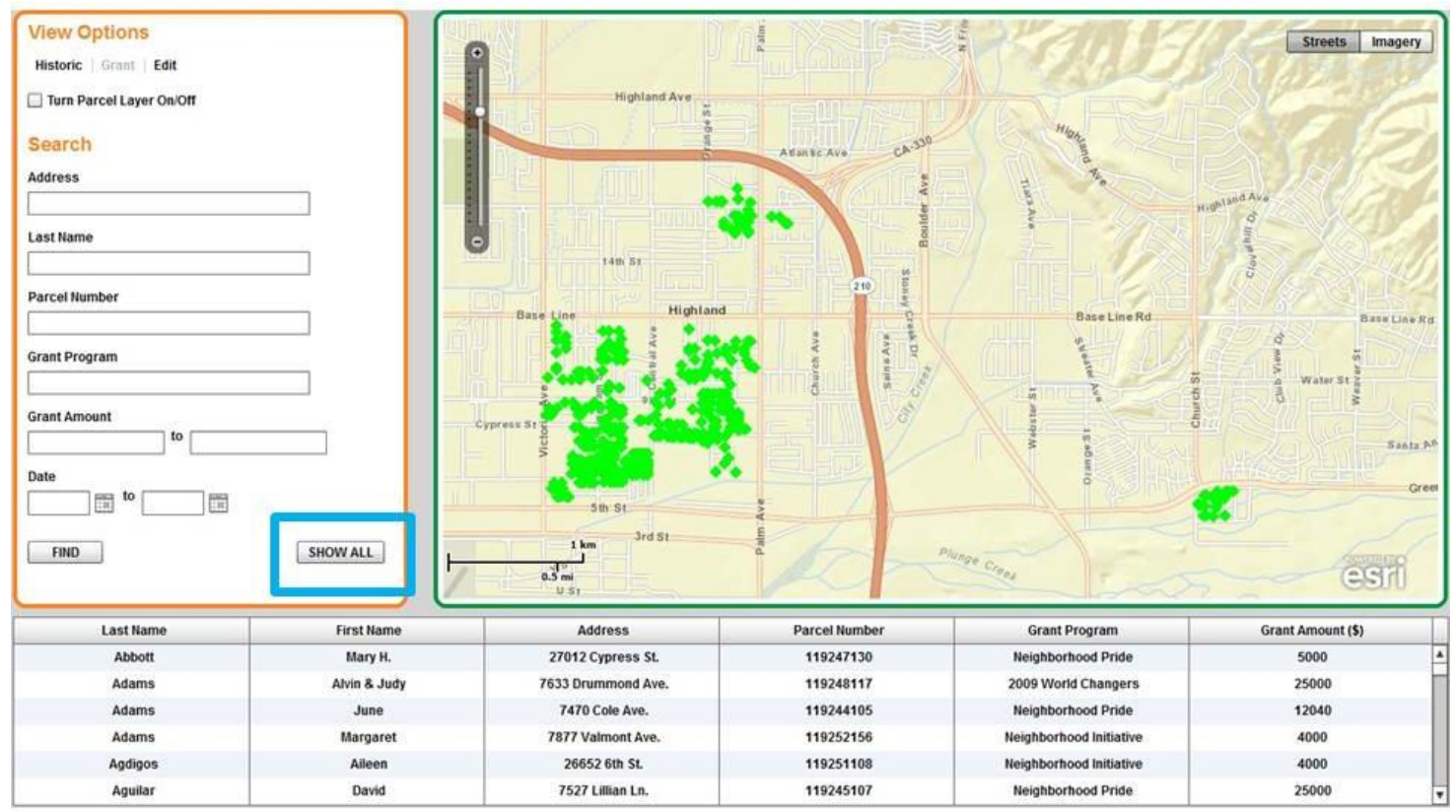

Figure 6-7: Return to Full Extent 


\subsection{Editing Data}

Now the Economic Department of Highland would like to add a historically designated building to their database. These new records are sent to the Economic Department for storage and maintenance. When the Department receives these new records, they want to add it to their database by using the web application. Once the application is launched, the user needs to switch to the Edit View using the View Options where he/she can find instructions on how to add a record to either of the datasets (Figure 6-8).

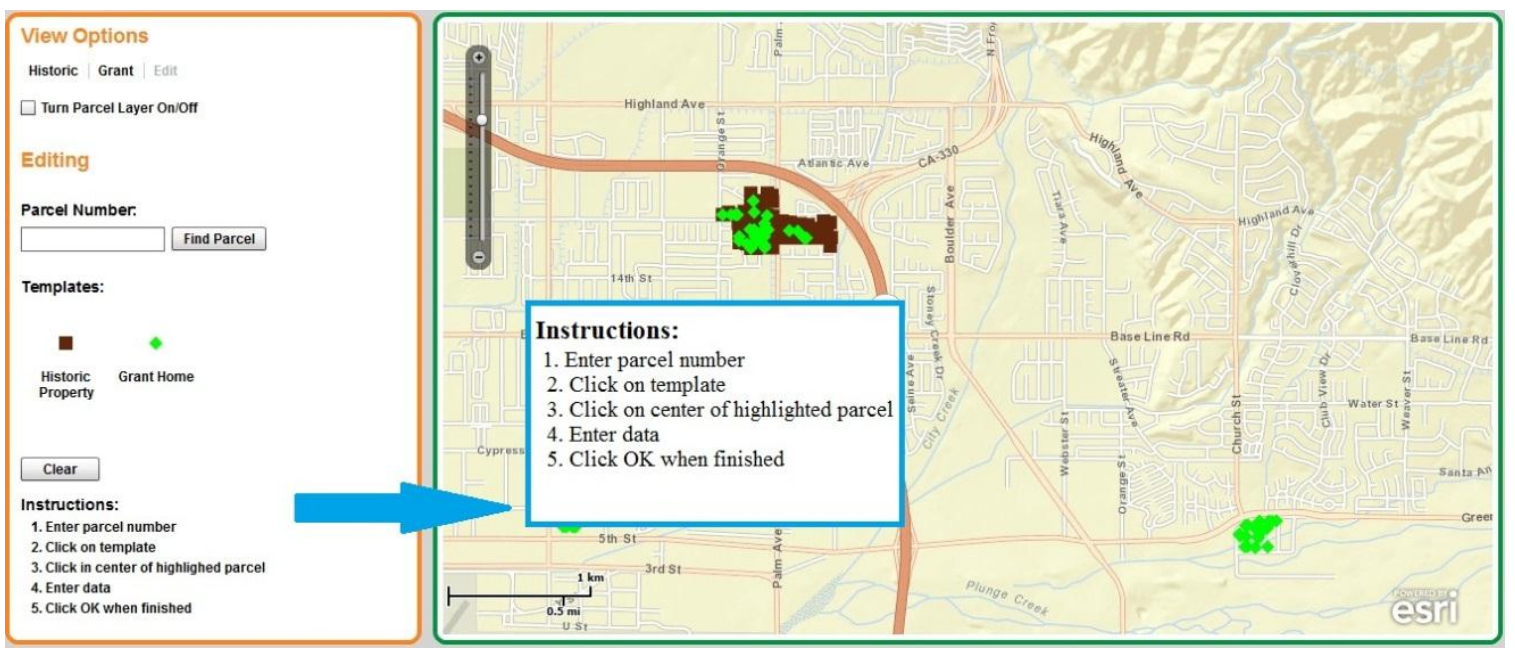

Figure 6-8: View Options

The first step in the editing process is to query for the parcel in which the new record's address is found. When the user clicks "Find Parcel", the query function zooms to the correct parcel and displays it in a graphics layer (Figure 6-9).

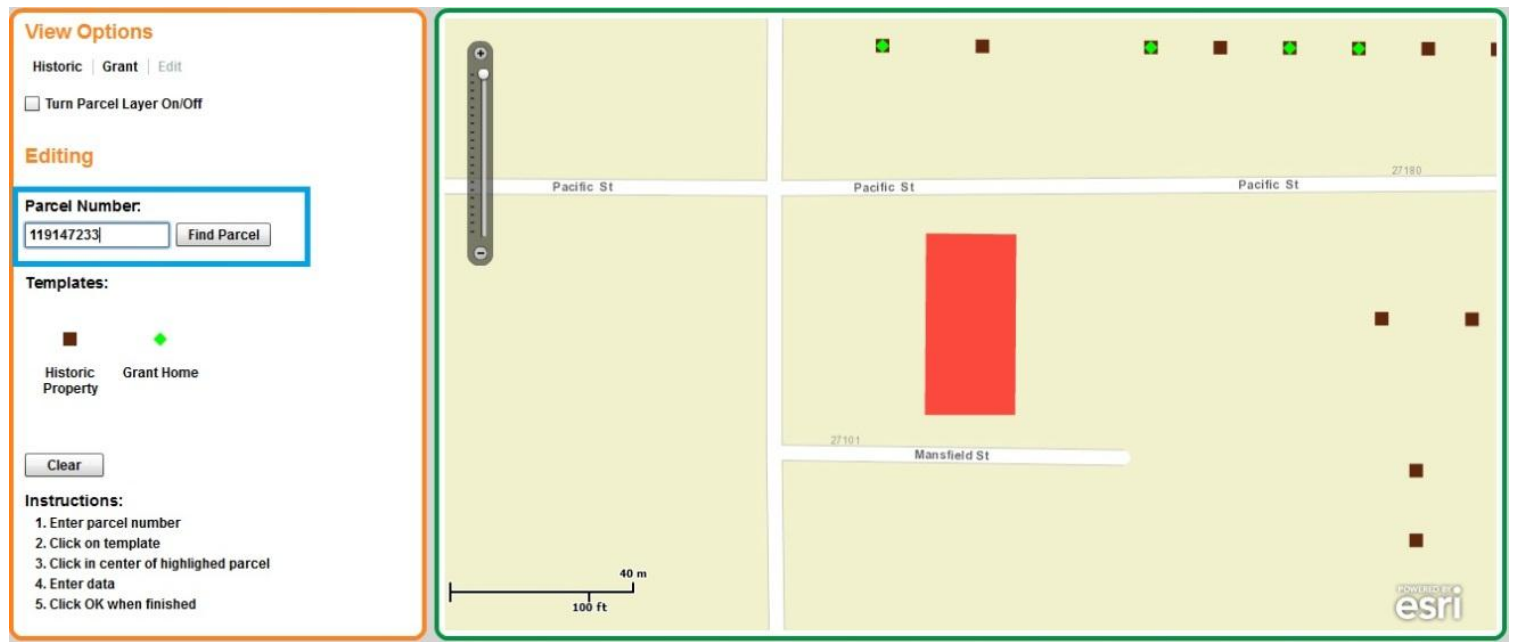

Figure 6-9: Finding Parcel for Editing Process

The user then selects the "Historic Property" template (highlighted in Figure 6-10) in the Templates section and place the new feature in the center of the highlighted parcel. This 
will open an Attribute Inspector where the new records attributes are entered (Figure 611).

\section{Templates:}

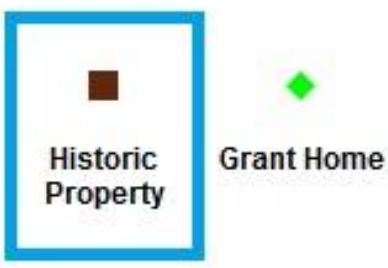

Figure 6-10: Editing Templates

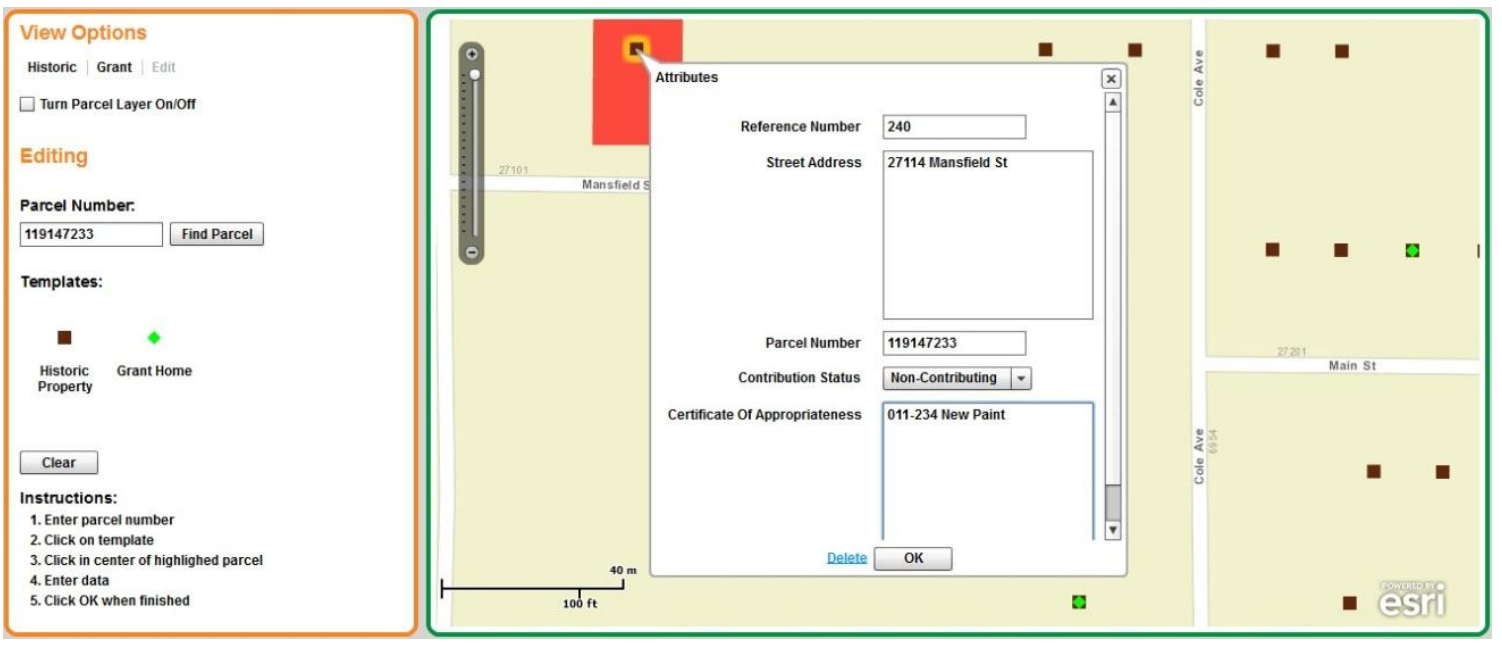

Figure 6-11: Attribute Editor

When the user clicks "OK" the attribute window closes and the new record is automatically added to the geodatabase. If the user had made a mistake, he/she can click "Delete" and the new feature will be deleted along with any entered attributes. When the edits are complete, the user can choose to move back to the Historic page. To view the new feature on the map, the "Show All" button refreshes the map and any new features will now be showing on the map and in the Results Table below (Figure 6-12). 


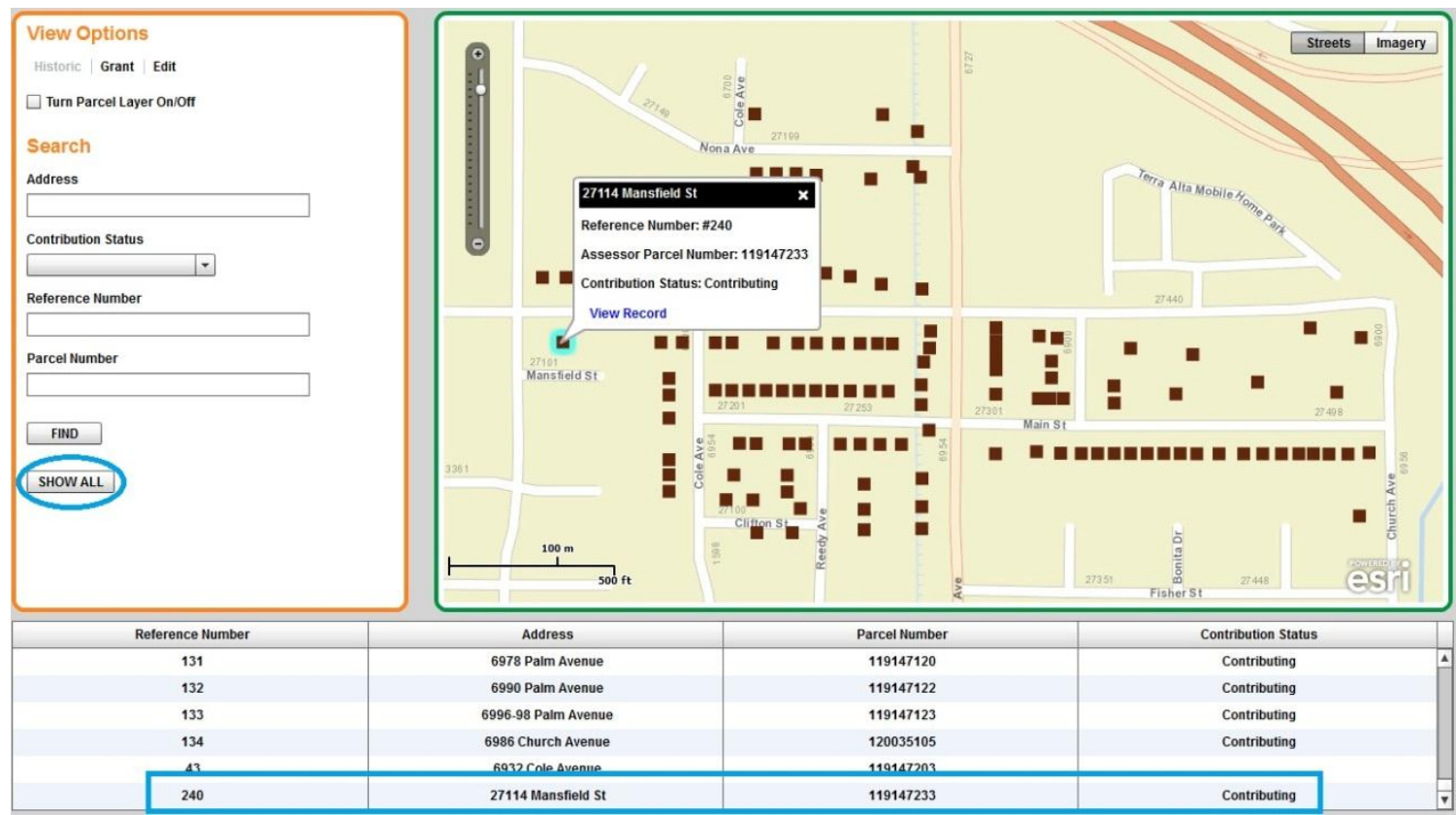

Figure 6-12: Feature Added

Unfortunately the staff who entered this record made a mistake when entering in the attributes of the new feature. The record is not "Non-Contributing"; it is "Contributing. They will move back to the Edit view and click on the point. This will reopen the Attribute Inspector window. They will change the Contribution Status to "Contributing" using the drop-down box (Figure 6-13). When moving back to the Historic view, the attribute will reflect the changes made (Figure 6-14).

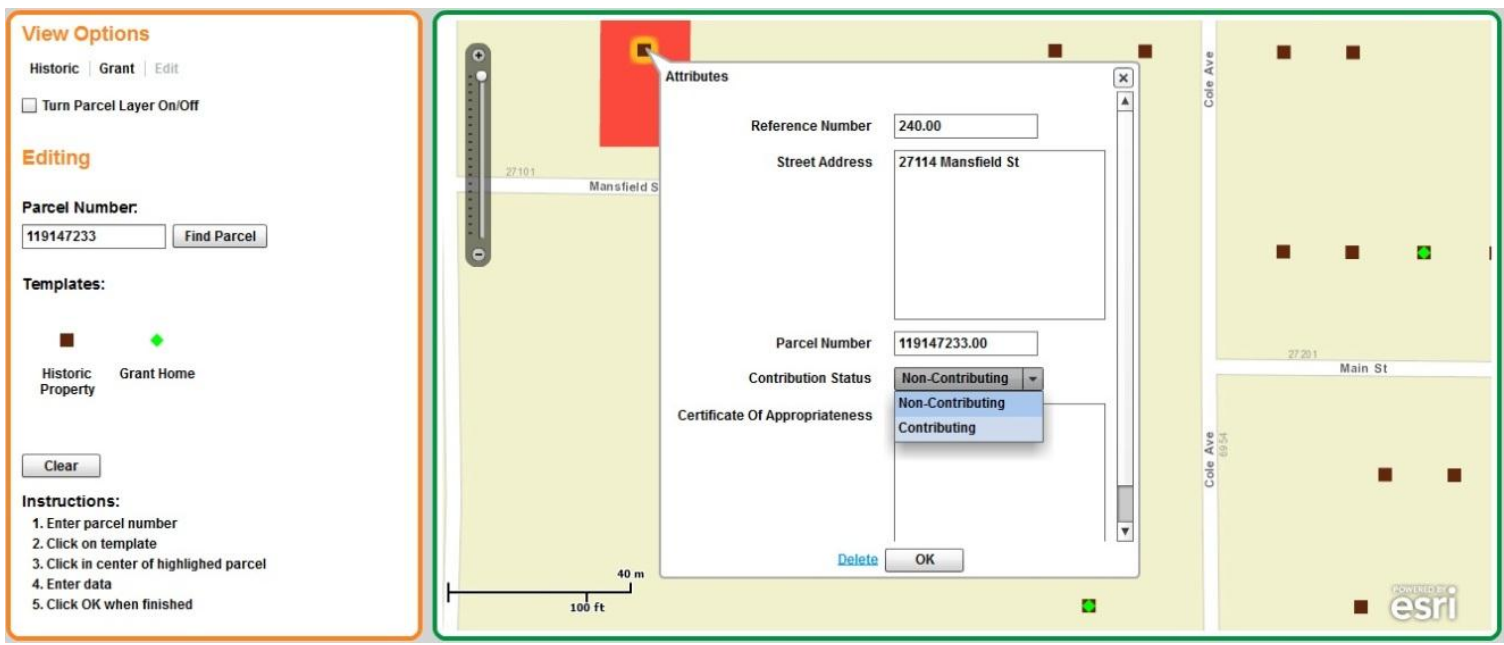

Figure 6-13: Editing Existing Attributes 


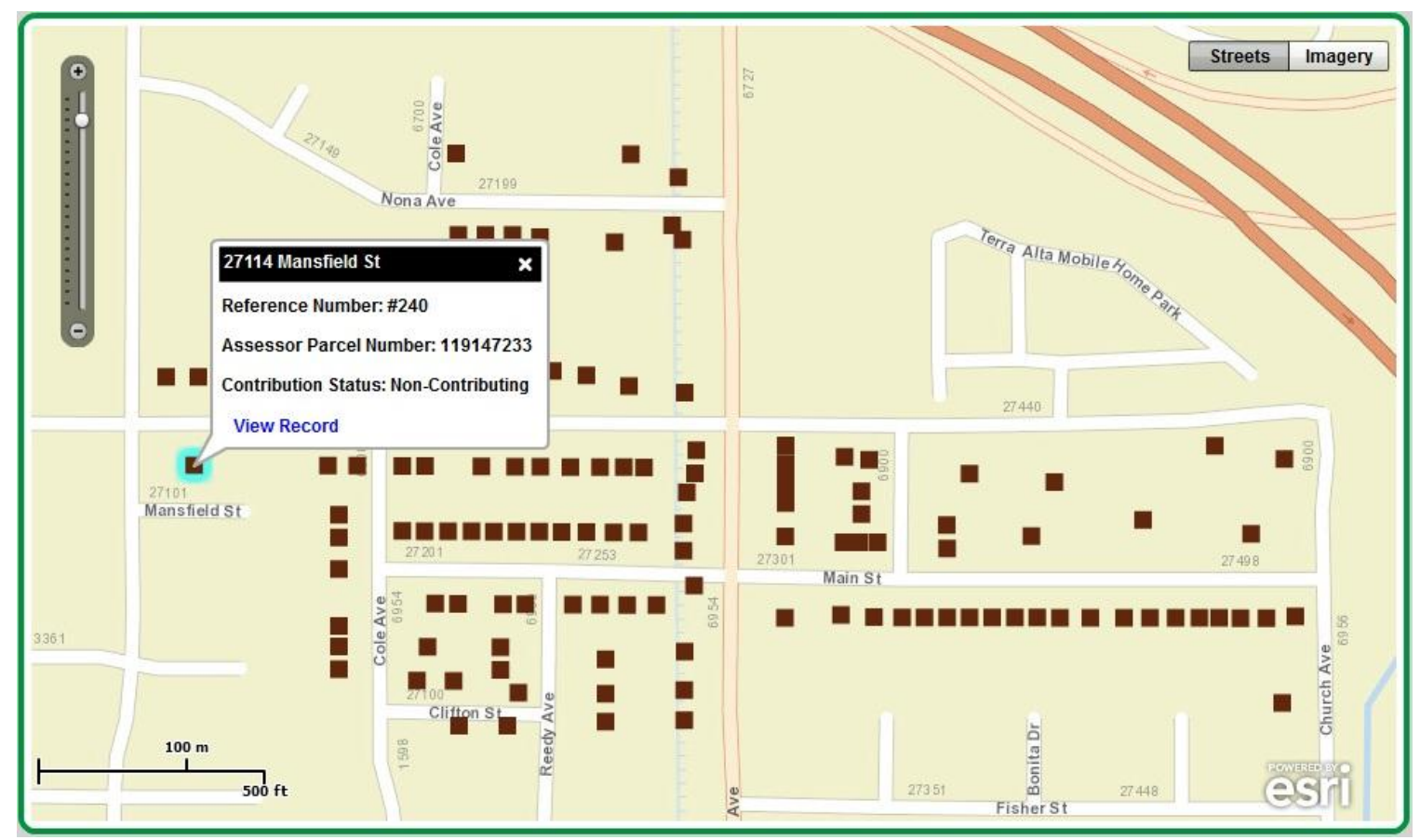

Figure 6-14: Edited Attributes

\subsection{Summary}

This chapter described the two primary uses for the web application that was built for the Economic Department of Highland, CA by giving two examples of use. The browsing and querying functions were described in a use case designed for the Building and Safety Department. They want to search for a record to determine if permit fees may be waived for work done by a contractor. The second use case described the process for the Economic Department to add a historically designated building. 


\section{Chapter 7 - Conclusions and Future Work}

This project was done for the Economic Department in Highland, California. They needed a way to easily store and update data concerning historically designated buildings and properties and homes that had been improved using grant money. They also wanted to be able to share their data with the other departments in the City. A proposed solution for this problem was a web application with query and edit capabilities. The goals of this project were the building of a geodatabase to store the client's data and the building of an intuitive web application to access this database. The web application was to be made available to the other departments in the City but not to the general public. These goals were used to measure the success of the project. Several needs were exposed during the requirements analysis portion of the project. These include an ArcSDE Geodatabase and ArcServer 10, as well as the ArcGIS API for Flex.

Data concerning historically designated buildings and homes that were improved using grant money were received from the client. Also used was parcel data from the San Bernardino Associated Governments. An ArcSDE Geodatabase was then built using these datasets. A map document was created using feature classes stored in this geodatabase and was published to the web using ArcServer 10.

The web application was built using the ArcGIS API for Flex and was written and complied in Adobe Flash Builder. Several functions were coded to customize the interface for the application. The user can click on each record and view attributes in an Info Window. For the historic data, an excerpt from the historical report can be viewed in its entirety in a new window by clicking on the "View Record" link. A search feature layer function was coded to allow the user to search for multiple attributes at the same time or even an incomplete attribute. The search results are displayed on the map and in a result table at the bottom of the page. The final functionality of the web application was the ability to edit the geodatabase through the interface. The user finds the parcel that will contain the new record then, using a template, places the new point on the map and enters the new attributes into a window that appears when the point is placed.

During the successful completion of this project, several ideas were thought of to further expand upon the work previously done. There are a few small areas in which this project could have been expanded, but due to budget or technology constraints they were not able to be completed. The first of these being photos of each historic building were to be included in the previously mentioned historical report. Secondly, the historic portion of the application could be viewed by the general public. This was changed due to technological uses with the client missing key software. Finally, a function or geoprocessing tool could be coded so that the editing process can take places all in one step. The user could simply enter the attributes into a form and then click save. The function would query the parcel number entered, find the location, and attach the additional entered attributes. In the final step it would apply the edits to the geodatabase and add the new point to the map.

Another area of expansion could be into analysis. Although the client did not need any analysis at this time, one question that could be asked of this data concerns neighborhood value. If home owners in a certain area are given grants to improve their homes, after the improvements, does this increase the overall value of the neighborhood? 
Do these improvements raise the value of homes that have not had the improvements? This would require the analysis of multiple years of housing prices of the neighborhoods in question.

Finally, another area to further develop this project would be to expand the web application to include data from all Highland's government departments, such as Community Development, or Building and Safety. The same portal could be used by multiple departments, with each of them having their own view and links being added to navigate between these views.

In conclusion, this project demonstrates how a simple, intuitive web application can be built to increase the workplace efficiency of a small city government. 


\section{Works Cited}

City of Highland Map. (2007). Retrieved from City of Highland: http://www.ci.highland.ca.us/Map/

Intelligent web maps and ArcGIS Online. (2011, Summer). Arc News.

SANBAG GIS Data. (2011). Retrieved from San Bernardino Associated Governments: http://maps.sanbag.ca.gov/website/misc.htm

Adnan, M., Singleton, A., \& Longley, P. (2010). Developing Efficient Web-based GIS Applications. Working Papers Series, 153, 1-15.

Anderson, E. (2005). Overview. In C. Fleming, The GIS Guide for Local Government Officials (pp. 1-7). Redlands: ESRI.

Baker, M., \& Jayaprakash, A. (2009). Building Rich Internet Applications with ArcGIS API for Flex. Redlands: Esri.

Brown, C. (2012). Getting Started with ArcGIS Web Mapping APIs. Esri California, Hawaii, Nevada Regional User Conference. Redlands: ESRI.

City of Highland. (2007). About the City. Retrieved from City of Highland: http://www.ci.highland.ca.us/AboutTheCity/

City of Riverside. (n.d.). Historic Districts \& Buildings. Retrieved from http://olmsted.riversideca.gov/historic/ppty_lkp.aspx

Dwyer, E. K. (2011). Chapter 7 - Ireland. In D. D. Wright, Coastal Informatics: Web Atlas Design and Implementation (pp. 105-130). Hershey: IGI Global.

Esri. (2010). 2010 Esri International User Conference $Q \&$ A. Retrieved April 24, 2012, from 2010 Esri International User Conference:

http://events.esri.com/uc/QandA/index.cfm?fuseaction=answer\&conferenceId=2F 6DC1A1-1422-2418-883C3868A9004888\&questionId=3061

Esri. (2011). ArcGIS API for Flex Code Examples. Retrieved from http://help.arcgis.com/en/webapi/flex/samples/index.html\#/InfoWindow_on_Clic k/01nq00000031000000/

Esri. (2011). Editing. Retrieved from ArcGIS API for Flex:

http://help.arcgis.com/en/webapi/flex/help/index.html\#/Editing/017p0000001800 0000/

Esri. (2012, 7 13). ArcGIS API for Silverlight overview. Retrieved from ArcGIS

Resources: http://resources.arcgis.com/en/help/silverlightapi/concepts/index.html\#//016600000006000000

Esri. (n.d.). Geodatabase: Multiuser Functionality. Retrieved from Esri Products: http://www.esri.com/software/arcgis/geodatabase/multi-user-functionality.html

Fu, P., \& Sun, J. (2011). Web GIS Principles and Applications. Redlands: Esri.

Kraak, M. (2004). The role of the map in a Web-GIS environment. Journal of Geographical Systems, 6, 83-93. doi:10.1007/s10109-004-0127-2

Li, S., Xiong, C., \& Ou, Z. (2011). A Web GIS for Sea Ice Information and an Ice Service Archive. Transactions in GIS, 15(2), 189-211. doi:10.1111/j.14679671.2011.01250.x

Mathiyalagan, V., Grunwald, S., Reddy, K., \& Bloom, S. (2005). A WebGIS and geodatabase for Florida's wetlands. Computers and Electronics in Agriculture, 47, 69-75. doi:10.1016/j.compag.2004.08.003 
O'Rourke, C. (2004, July/August). A Look at Rich Internet Applications. Retrieved from Oracle Magazine: http://www.docstoc.com/docs/85265716/Dev-Trends_-A-Lookat-Rich-Internet-Applications

Zeiler, M. (2010). Modeling Our World. Redlands: Esri. 


\section{Appendix A. Web Map Application Code}

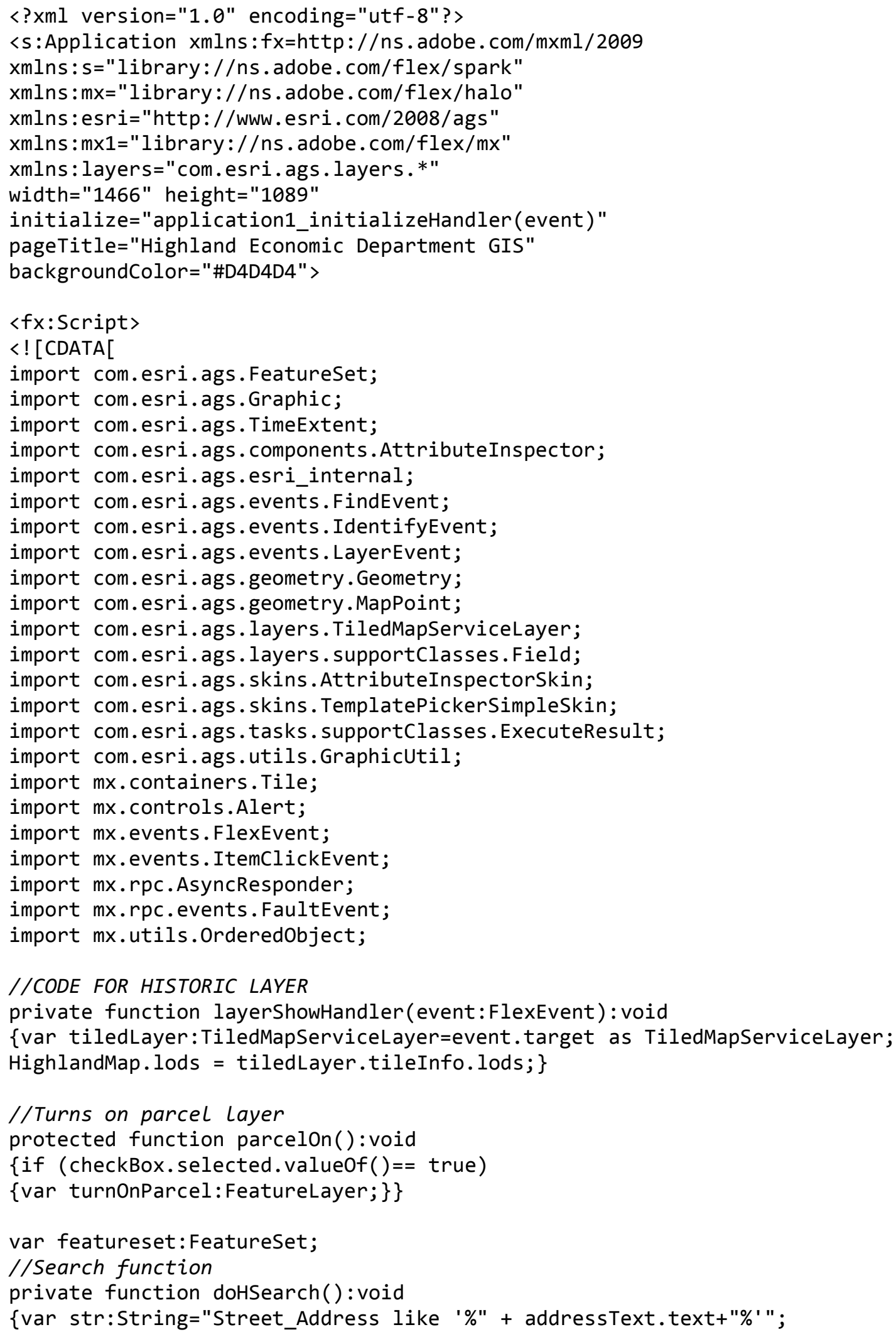




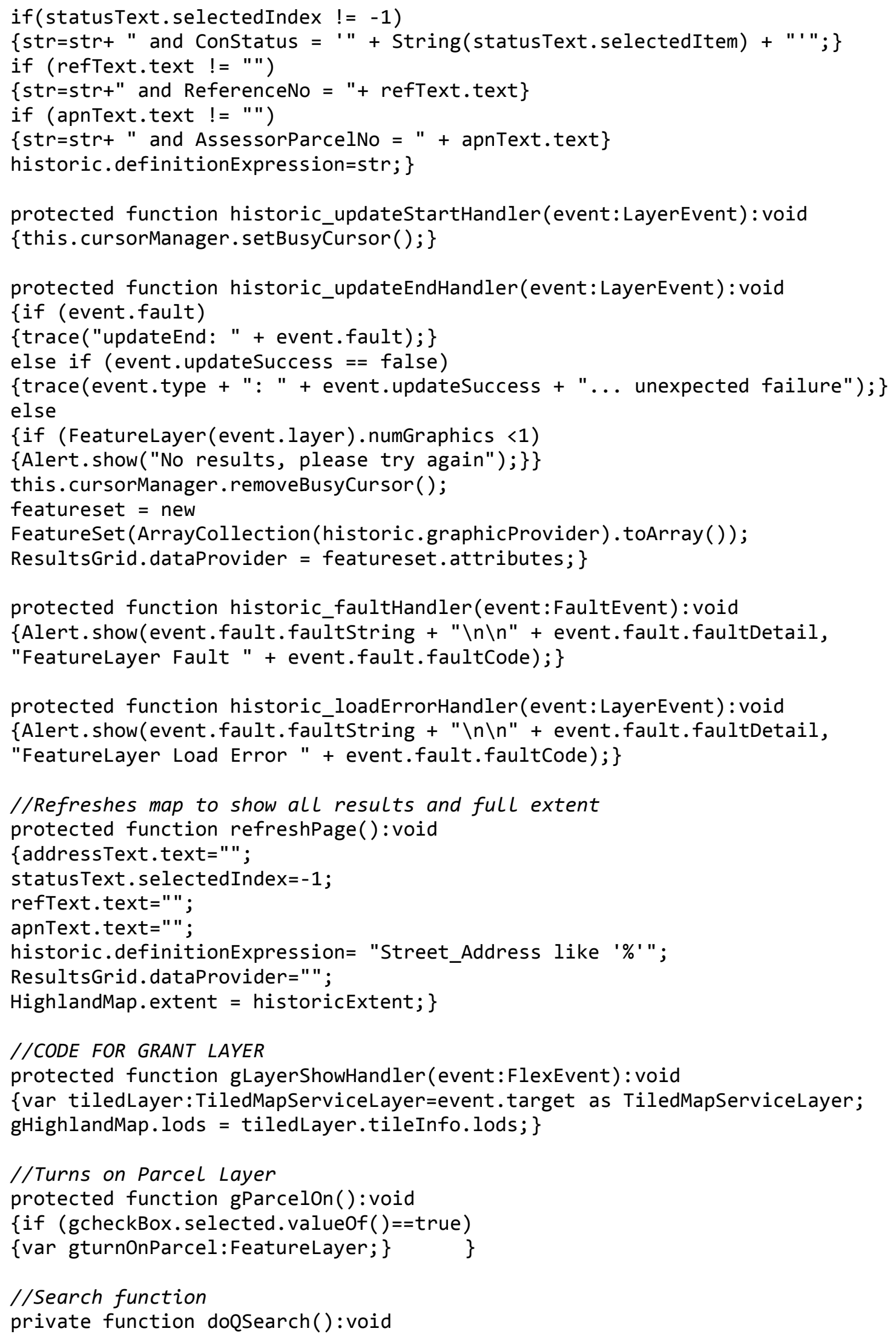


$\{$ var str:String = "Street_Address like '\%" + gaddressText.text + "\%'";

if (gnameText.text != "")

$\{$ str $=$ str + " and LName like '\%" + gnameText.text + "\%'"\}

if (gpnText.text != "")

$\{$ str $=$ str $+"$ and Parcel_Number $=' "+$ gpnText.text $\}$

if (gprogramText.text != "")

\{str = str + " and Grant_Program like '\%" + gprogramText.text + "\%'"\}

if (grantAmount1.text !="")

$\{$ str $=$ str + " and Grant_Amount $>="+$ grantAmount1.text $\}$

if (grantAmount2.text !="")

\{str = str + " and Grant_Amount $<="+$ grantAmount2.text $\}$

if (date1.text != "" \&\& date2.text!="")

$\{$ grant.timeDefinition $=$ new TimeExtent (date1.selectedDate, date2. selectedDate);

grant.definitionExpression=str;

protected function grant_updateStartHandler(event:LayerEvent): void

\{this.cursorManager.setBusyCursor ( );

protected function grant_updateEndHandler(event:LayerEvent): void

\{if (event.fault)

\{trace ("updateEnd: " + event.fault); \}

else if (event. updateSuccess $==$ false)

\{trace (event.type $+":$ " + event.updateSuccess $+" \ldots$ unexpected failure");

else

\{if (FeatureLayer (event.layer).numGraphics <1)

\{Alert.show("No results, please try again"); \}

this.cursorManager. removeBusyCursor();

featureset $=$ new FeatureSet (ArrayCollection(grant.graphicProvider).toArray ()$)$;

gResultsGrid.dataProvider = featureset. attributes; $\}$

protected function grant_faultHandler(event:FaultEvent): void

\{Alert.show(event.fault.faultString + "\n\n" + event.fault.faultDetail,

"FeatureLayer Fault " + event.fault.fault(Code);

protected function grant_loadErrorHandler(event:LayerEvent):void

\{Alert.show(event.fault.faultstring + "\n\n" + event.fault.faultDetail,

"FeatureLayer Load Error " + event.fault.faultCode);\}

//Refreshes map to show all results and full extent

protected function showAll():void

\{gaddressText.text=" ";

gnameText.text=" ";

gpnText.text=" ";

gprogramText.text=" ;

grant.definitionExpression="Street_Address like '\%'";

gResultsGrid.dataProvider=" ";

date1.text="";

date2.text=" ";

gHighlandMap.extent = grantExtent;

grant.timeDefinition= new TimeExtent (new Date("01/01/1990 1:00:00"), new

Date("01/01/3000 1:00:00"));

//CODE FOR EDITING

protected function eParcelon(): void

\{if (echeckBox. selected. valueOf ()$==$ true) 
$\{$ var eturnOnParcel:FeatureLayer; $\}$ \}

protected function doQuery(): void

\{graphicsLayer.clear();

queryTask.execute(query, new AsyncResponder(onResult, onFault));

function onResult (featureSet:FeatureSet, token:Object $=$ null): void

$\{$ if (featureset.features. length $==0$ )

\{Alert.show("Parcel not found, try again");

else

$\{$ var graphicsExtent: Extent $=$

GraphicUtil.getGraphicsExtent(featureset.features);

if (graphicsExtent)

$\{$ eHighlandMap.extent $=$ graphicsExtent; $\}\}\}$

function onfault (info:Object, token:Object $=$ null): void

\{Alert.show(info.tostring ()); \}

protected function application1_initializeHandler(event:FlexEvent): void

\{myEditor.featureLayers $=$ [historicEdit, grantEdit]; $\}$

protected function clearParcel():void

\{parcelText.text=" ";

graphicsLayer.clear();

eHighlandMap.extent $=$ editExtent; $\}$

]]>

$\langle/$ fx:Script $\rangle$

$\langle\mathrm{fx}:$ Style $>$

@namespace s "library://ns.adobe.com/flex/spark";

@namespace mx "library://ns.adobe.com/flex/mx";

@namespace esri "http://www.esri.com/2008/ags";

esri|Infowindow

\{background-color: \#FFFFFF; border-thickness: 2 ; $\}$

esri|TemplatePicker

\{skinClass:ClassReference("com.esri.ags.skins.TemplatePickerSimpleSkin");

$\langle/$ fx:Style $>$

$\langle f x$ :Declarations>

<s:DateTimeFormatter id="dtfInfoWindow" dateTimePattern="MMMM dd, yyyy"/>

<esri:Simplefillsymbol id="sfs" alpha="0.7" color="0xFF0000"/>

<esri:QueryTask id="queryTask"

url=http://msgis-ags-1/ArcGIS/rest/services/C20-

amanda_hutsel/HighlandMapSDE3/MapServer/2 useAMF="false"/>

<esri:Query id="query" returnGeometry="true"

outSpatialReference $=$ " $\{$ eHighlandMap. spatialReference $\}$ " where="APN =

\{parcelText.text\}"/>

<esri:GeometryService id="myGeometryService" url="http://msgis-ags-

1/ArcGIS/services/C20-amanda_hutsel/Geometry/GeometryServer"/>

$\langle/$ fx:Declarations $\rangle$

$<$ !--BANNER \& ViewStack-->

<s:BitmapImage id="HighlandBanner" horizontalCenter="0" top="3" scaleX="1"

scaleY="1" source="@Embed('images/doubleBanner.jpg')"/>

$\langle m \times 1$ :ViewStack id="myViewStack" creationPolicy="all"> 


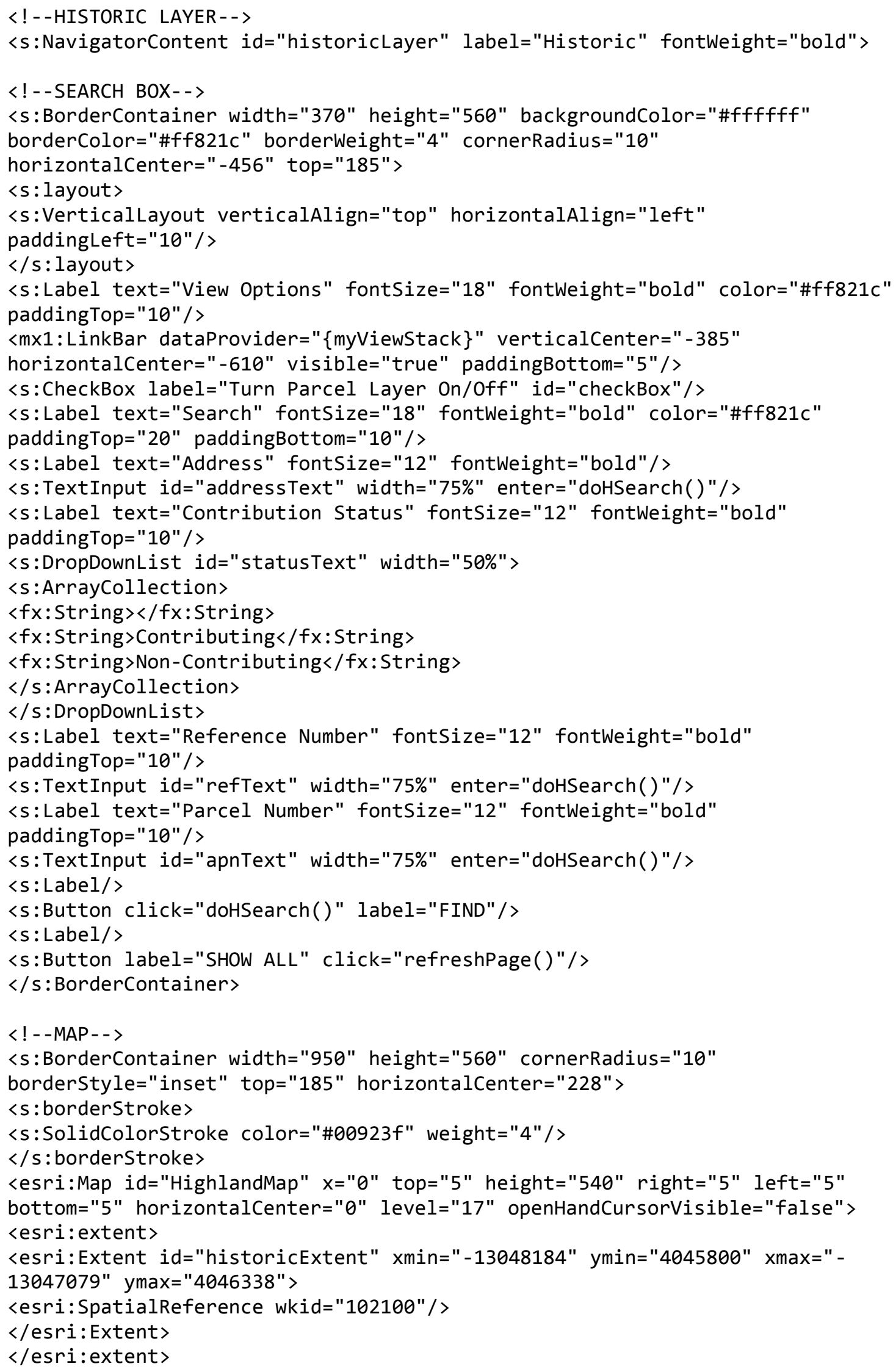


<esri:ArcGISTiledMapServiceLayer show="layerShowHandler(event)"

url=http://server.arcgisonline.com/ArcGIS/rest/services/World_Street_Map/MapSe rver visible $=$ " ButtonBar. selectedIndex $==0\} " />$

<esri:ArcGISTiledMapServiceLayer show="layerShowHandler(event)"

url=http://server.arcgisonline.com/ArcGIS/rest/services/World_Imagery/MapServe

$r$ visible $="\{$ ButtonBar. selectedIndex $==1\} " />$

<esri:FeatureLayer url=http://msgis-ags-1/ArcGIS/rest/services/C20-

amanda_hutsel/HighlandMapSDE3/FeatureServer/2 show="parcelOn()"

visible $="$ checkBox. selected $==$ true $\} " />$

<esri:FeatureLayer id="historic" fault="historic_faultHandler(event)"

mode="snapshot" updateEnd="historic_updateEndHandler(event)"

updateStart="historic_updateStartHandler(event)"

outFields="[ReferenceNo, Street_Address, AssessorParcelNo, Constatus,

CertificateOfAppropriateness, Links]"

url="http://msgis-ags-1/ArcGIS/rest/services/C20-

amanda_hutsel/HighlandMapSDE3/FeatureServer/0">

〈esri:infowindowRenderer>

$\langle\mathrm{fx}$ :Component>

$<m \times 1:$ VBox label="\{data.Street_Address\}" borderColor="\#00923f"

backgroundColor="\#ffffff">

$\langle$ fx:Script $\rangle$

$<![$ CDATA [

import flash.net.navigateToURL;

protected function linkToHistory (): void

\{var theHistoryPage:URLRequest = new URLRequest(data.Links);

navigateToURL(theHistoryPage, "_blank");\}]] >

$\langle/$ fx:Script $\rangle$

$\langle m \times 1$ :Label text="Reference Number: \#\{data.ReferenceNo\}"/>

<mx1:Label text="Assessor Parcel Number: \{data.AssessorParcelNo\}"/>

$<m \times 1$ :Label text="Contribution Status: \{data.ConStatus\}"/>

$\langle m \times 1$ :LinkButton label="View Record" color="blue" click="linkToHistory()"/>

$\langle/ m \times 1:$ VBox $>$

$\langle/$ fx:Component $\rangle$

</esri:infoWindowRenderer>

<esri:renderer>

〈esri:UniqueValueRenderer>

〈esri:defaultSymbol>

<esri:SimpleMarkerSymbol color="\#61280E" style="square" size="12"/>

$\langle/$ esri:defaultSymbol>

$</$ esri:UniqueValueRenderer >

$\langle/ e s r i:$ renderer $>$

$\langle/$ esri:FeatureLayer >

$</$ esri:Map>

$\langle$ !--Switch between basemaps-->

<s:ButtonBar id="ButtonBar" right="10" top="15" requireSelection="true">

$\langle s$ :dataProvider >

〈s:ArrayList>

$\langle f x$ :String $>$ Streets $</ f x$ :String $>$

$\langle\mathrm{fx}$ :String $>$ Imagery $\langle/ \mathrm{fx}$ :String $\rangle$

$\langle/ \mathrm{s}$ :ArrayList $\rangle$

$\langle/ \mathrm{s}$ : dataProvider $>$

$\langle/ \mathrm{s}$ : ButtonBar $>$

$\langle/ \mathrm{s}$ :BorderContainer $\rangle$ 
$\langle!--$ RESULTS GRID-->

<mx1:DataGrid id="ResultsGrid" x="63" y="753" width="1345" textAlign="center" verticalAlign="middle" $\rangle$

$\langle m \times 1$ : columns>

<mx1:DataGridColumn dataField="ReferenceNo" headerText="Reference Number"/>

<mx1:DataGridColumn dataField="Street_Address" headerText="Address"/>

<mx1:DataGridColumn dataField="AssessorParcelNo" headerText="Parcel Number"/>

$\langle m \times 1$ :DataGridColumn dataField="ConStatus" headerText="Contribution Status"/>

$\langle/ m \times 1$ : columns $\rangle$

$</ m \times 1:$ DataGrid $>$

$\langle/ \mathrm{s}$ : NavigatorContent $\rangle$

$\langle$ !--GRANT LAYER-->

〈S:NavigatorContent id="grantLayer" label="Grant" fontWeight="bold">

$\langle!--$ SEARCH BOX -->

<s:BorderContainer $\mathrm{y}=$ "185" width="370" height="560" backgroundColor="\#ffffff" borderWeight="4" borderColor="\#ff821c" cornerRadius="10" horizontalCenter=" 456" top="185">

$\langle\mathrm{s}:$ layout $>$

$<s: V e r t i c a l L a y o u t$ verticalAlign="top" horizontalAlign="left"

paddingLeft="10" / >

$\langle/ \mathrm{s}:$ layout $\rangle$

$<\mathrm{s}$ :Label text="View Options" fontSize="18" fontWeight="bold" color="\#ff821c" paddingTop="10"/>

$<m \times 1$ : LinkBar dataProvider=" \{myViewStack $\}$ " verticalCenter=" -385"

horizontalCenter="-610" visible="true" paddingBottom="5"/>

<s:CheckBox label="Turn Parcel Layer On/Off" id="gcheckBox"/>

<s:Label text="Search" fontSize="18" fontWeight="bold" color="\#ff821c"

paddingTop $=" 20 "$ paddingBottom $=" 10 " />$

<s:Label text="Address" fontSize="12" fontWeight="bold"/>

<s:TextInput id="gaddressText" width="75\%" enter="doQSearch()" / >

<s:Label text="Last Name" fontSize="12" fontWeight="bold" paddingTop="10"/>

<s:TextInput id="gnameText" width="75\%" enter="doQSearch()"/>

<s:Label text="Parcel Number" fontSize="12" fontWeight="bold"

paddingTop $=" 10 " />$

<s:TextInput id="gpnText" width="75\%" enter="doQSearch()"/>

<s:Label text="Grant Program" fontSize="12" fontWeight="bold"

paddingTop $=" 10 " />$

<s:TextInput id="gprogramText" width="75\%" enter="doQSearch()" / >

<s:Label text="Grant Amount" fontSize="12" fontWeight="bold" paddingTop="10"/>

$\langle s:$ HGroup>

<s:TextInput id="grantAmount1" enter="doQSearch()" width="75\%"/>

$\langle s:$ Label text="to"/>

$\langle$ S:TextInput id="grantAmount2" enter="doQSearch()" width="75\%"/>

$\langle/ \mathrm{s}:$ HGroup $\rangle$

<s:Label text="Date" fontSize="12" fontWeight="bold" paddingTop="10"/>

$\langle\mathrm{S}:$ HGroup $\rangle$

$\langle m \times 1:$ DateField id="date1" formatString="MM-DD-YY"/>

$\langle$ s:Label text="to"/>

$\langle m \times 1$ :DateField id="date2" formatString="MM-DD-YY"/>

$\langle/ \mathrm{s}:$ HGroup $\rangle$

$\langle\mathrm{s}:$ Label/>

$\langle\mathrm{s}:$ HGroup> 


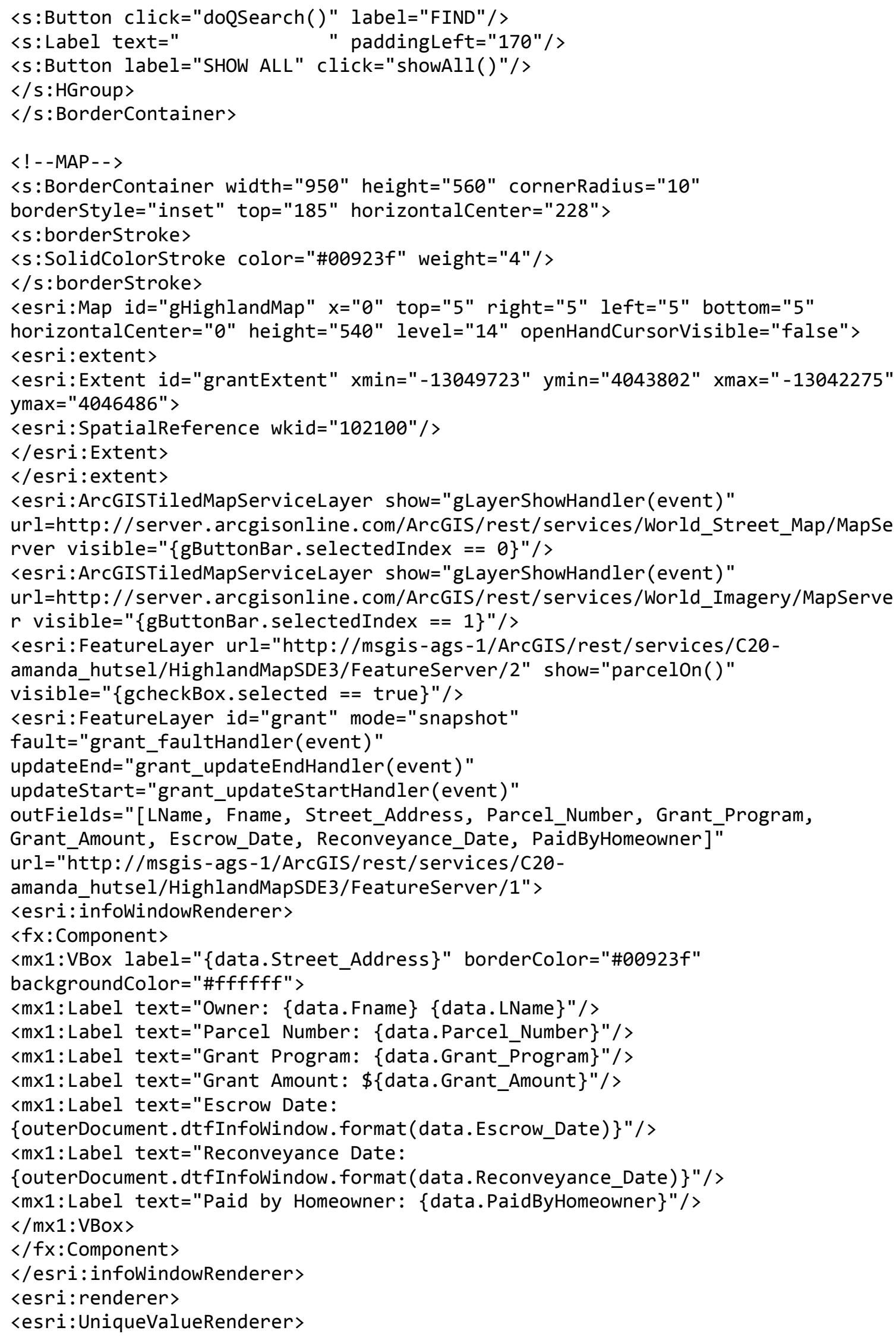




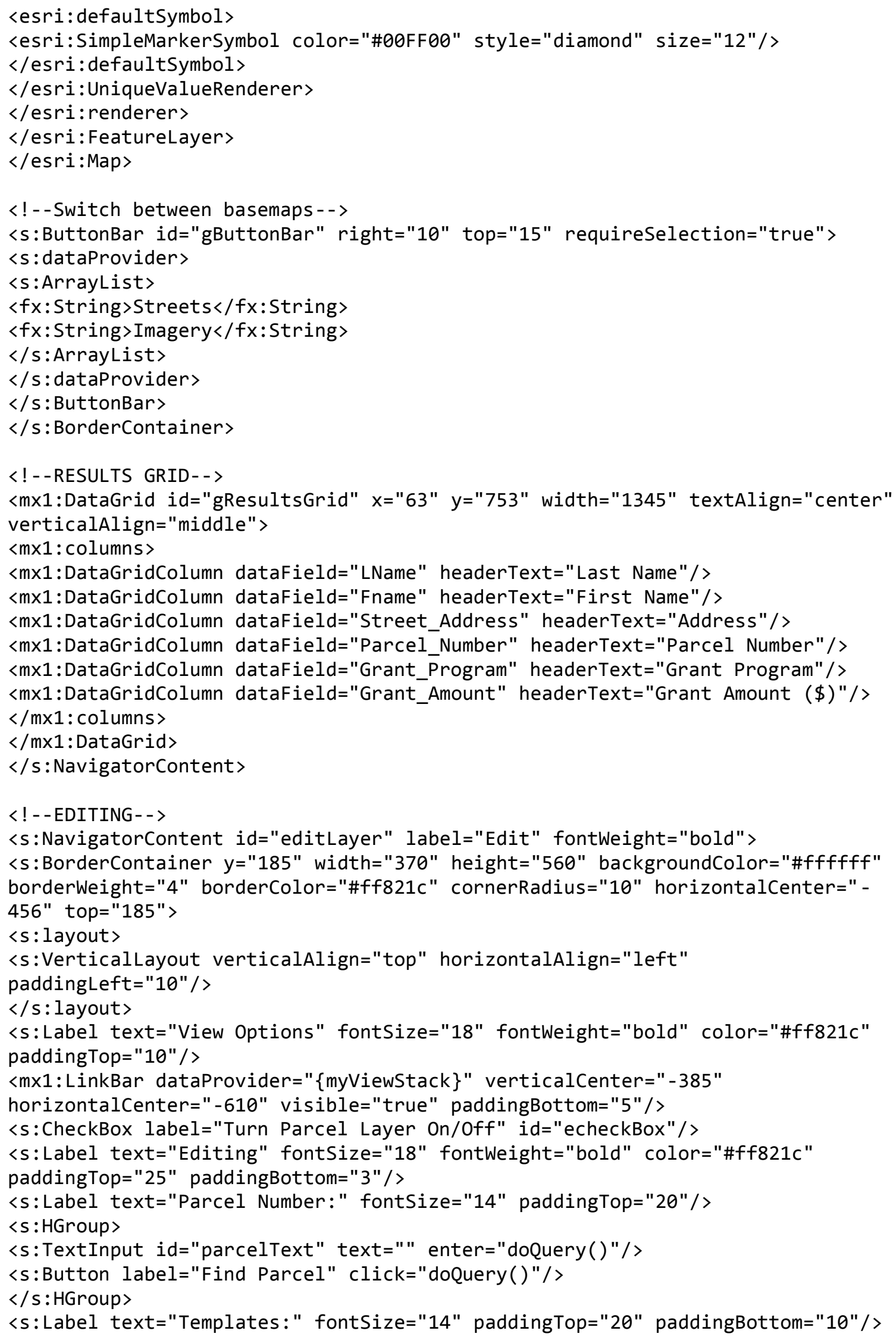




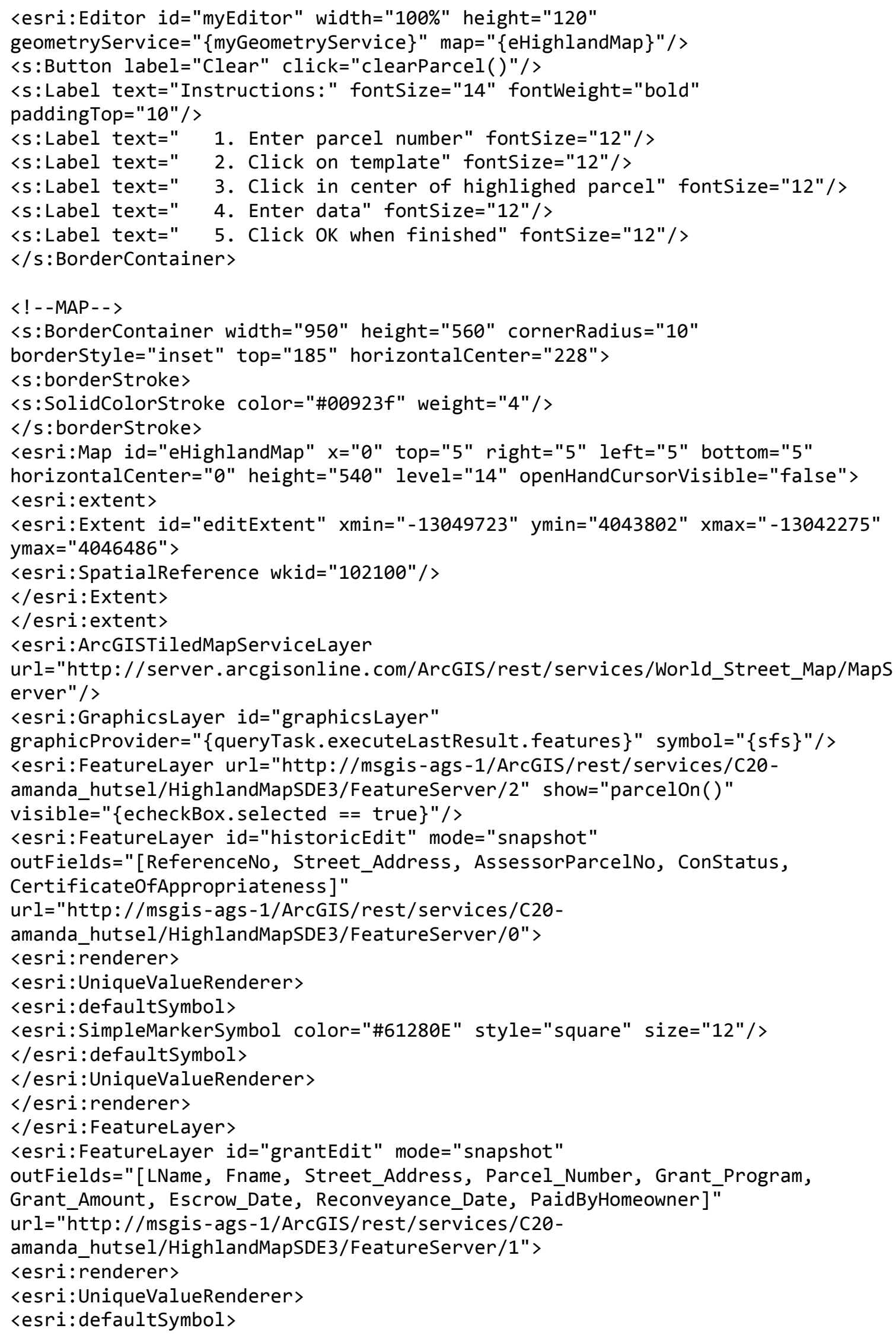




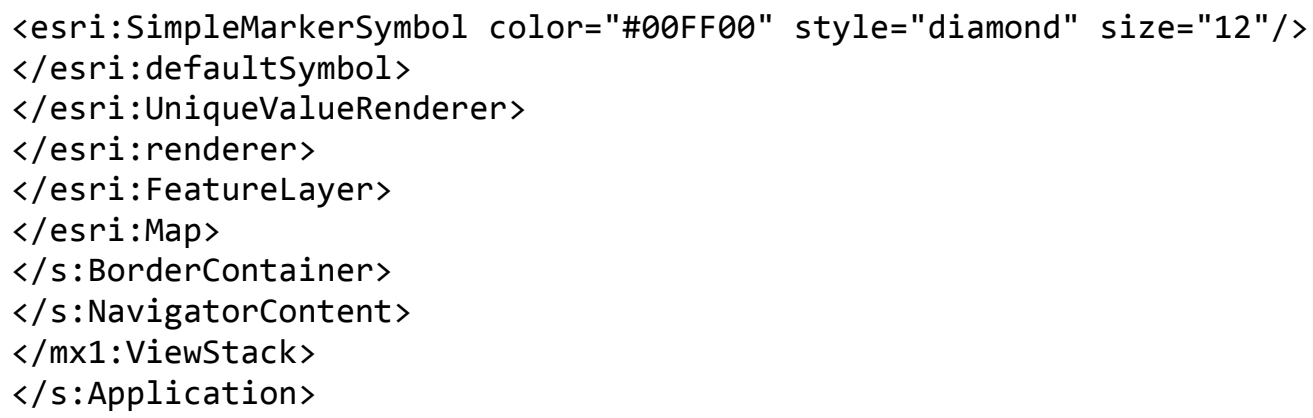

